\title{
Numerical Study on Dynamics and Polarization of the Hot Plasma Torus in the Magnetosphere: Cause of Generation of the Paired Region 1 and Region 2 Field-Aligned Currents
}

\author{
Takashi Yamamoto ${ }^{1}$, S. INOUE ${ }^{2}$, and C.-I. $\mathrm{MeNG}^{3}$ \\ ${ }^{1}$ Department of Earth and Planetary Physics, University of Tokyo, Tokyo 113, Japan \\ ${ }^{2}$ Aichi College of Technology, Gamagohri-shi, Aichi 443, Japan \\ ${ }^{3}$ The Johns Hopkins University, Applied Physics Laboratory, Laurel, Maryland 20723-6099, U.S.A.
}

(Received June 12, 1996; Revised December 28, 1996; Accepted April 3, 1997)

\begin{abstract}
To begin with, we postulate that in the magnetosphere, the hot ( $\gtrsim 1 \mathrm{keV})$ plasma particles are primarily distributed in a magnetic shell which is connected to two ovals of diffuse auroras on the northern and southern polar ionospheres. Such hot plasma population is called the hot plasma torus (HPT). To study dynamics and polarization of the HPT in the magnetosphere, we perform the two-dimensional numerical simulations which is capable of treating the electrostatic coupling between the magnetosphere and the ionosphere. The simulation results show that distortion of the HPT occurs due to the solar wind convection when the interplanetary magnetic field (IMF) is southward, so that the polarization of the distorted HPT produces a pair of the region 1 and region 2 field-aligned currents (FACs) which agrees with the observations. It is also shown that the self-distortion of the HPT (positive feedback) is possible; namely, the HPT is further polarized by electric fields arising from the polarized HPT, which leads to intensification of the region 1/region 2 FACs. Notably, in our model the disruption of a tail current is a natural result of the HPT distortion on the nightside, which can explain the observations that the tail current disruption occurs over a wide MLT range and well inside the magnetically closed region.
\end{abstract}

\section{Introduction}

Since the statistical characteristics of large-scale field-aligned currents (FACs) were summarized by Iijima and Potemra (1976), the region 1 FAC had been believed to be connected to a single topological region in the magnetosphere. However, Friis-Christensen and Lassen (1991) showed that the region 1 current consists of two separate regions, one (region 1a) connected to the low-latitude boundary layer (LLBL) and one (region 1b) connected to the plasma sheet. The recent data analysis of the magnetic field perturbation and the particle flux measured from the Defense Meteorological Satellite Program (DMSP) F7 satellite (Iijima et al., 1991; Iijima et al., 1992; Watanabe and Iijima, 1993) has shown that the region 1b FAC invariably is collocated with the region 2 FAC at lower latitudes, and that the paired region 1 and region 2 FACs coexist with enhanced precipitation of hot $(\gtrsim 1 \mathrm{keV})$ particles. In the present paper the combined system of the region $1 \mathrm{~b}$ and region 2 FACs is referred to as the paired region 1 /region 2 FACs, and the coexisting hot particle population in the magnetosphere is called the hot plasma torus (HPT), which can be regarded as the source of the paired region 1/region 2 FACs. Specifically, the HPT is the combined regions of the boundary plasma sheet (BPS) and the central plasma sheet (CPS). (The observations on the FAC system confined to the dayside are briefly reviewed by Yamamoto et al. (1996).)

As is discussed by Yamamoto et al. (1996), the persistent coupling feature of the paired region 1/region 2 FACs could not be understood within a frame of the traditional concepts: 1 . magnetic reconnection (Dungey, 1961), 2. solar wind-magnetosphere viscous interaction (Axford 
and Hines, 1961; Sonnerup, 1980), 3. ion inertial effect (Rostoker and Boström, 1976; Stern, 1983), 4. midnight-directed pressure gradient in the inner plasma sheet (Piddington, 1968; Wolf, 1983), and 5. cross-tail pressure gradient toward the tail flank in the outer plasma sheet (Karty et al., 1984; Yang et al., 1994). Yamamoto et al. study a theoretical model that the paired region 1/region 2 FACs can be generated as a result of "natural distortion" of the HPT due to the solar wind convection: owing to the distortion, the pressure gradient in the HPT gains a component parallel to the magnetic drift so that the particle magnetic drifts induce the polarization of the HPT with the polarity consistent with the observed pattern of the region 1 /region 2 FACs. (The magnetic drift means the gradient $B$ drift plus the curvature drift for charged particles.) In this model at any time the region 1 and region 2 FACs are generated simultaneously, but they are not balanced in intensity. (The ratio of the region 2 current intensity to the region 1 intensity, $I_{2} / I_{1}$, is presumed to be less than unity.)

The purpose of the present paper is to explicitly show, by using the two-dimensional (on the ionospheric plane) numerical simulations, that the paired region 1 /region 2 FACs are generated as a result of the HPT distortion under the influence of the solar wind convection (when IMF $\left.B_{Z}<0\right)$. Moreover, by including an effect of the anomalous cross-field diffusion on the HPT polarization, we can reproduce the triple FAC structure (i.e., upward FAC zone sandwiched between two downward FAC zones) in the midnight or premidnight sector to find a total FAC pattern in good agreement with the observations (Yamamoto et al., 1997b). The numerical simulations in the present paper also show that the self-distortion of the HPT (positive feedback) is possible; namely, the HPT is further polarized by electric fields arising from the polarized HPT, which leads to intensification of the paired region 1/region 2 FACs. From the simulation results it is found that the ratio of the region 2 current intensity to the region 1 intensity, i.e., $I_{2} / I_{1}$ is less than unity. The FAC generation with $I_{2} / I_{1}<1$, on the nightside, implies the disruption of tail currents. From this finding we can physically understand the observations that the tail current disruption occurs over a wide MLT range and well inside the magnetically closed region. A key to the generation of the paired region 1 and region 2 FACs is the existence of the HPT which is characterized by the latitudinal profile of the flux tube content $N$ (not the "local" number density $n$ ) showing a peak inside the closed region. (For the definition of $N$, see the next section.) When a hot plasma is distributed in such a way that $N$ is overall uniform poleward of the inner edge of the hot plasma domain, $n$ is peaked at the inner edge and it has an equatorward gradient inside the plasma domain. In this case, however, the region 1 FAC does not occur even if the hot plasma is deformed by the convection. This is the case with the (earlier version) simulation of the Rice Convection Model (e.g., Harel et al., 1981a, 1981b; Wolf, 1983). In that simulation model, the region 2 FACs were generated near the inner edge of the deformed hot plasma domain, by the same mechanism as in our model, namely by the azimuthal pressure gradient (see Fig. 6 of Wolf (1983)); the appearance of FACs of the region 1 sense (see Figs. 12 and 16 of Wolf) was required for a balance with the produced region 2 FACs (i.e., total current closure), but they were not self-consistently produced by a generator in the magnetosphere. The advanced version of the RCM (Karty et al., 1984; Yang et al., 1994) treated the non-uniform distribution of the flux tube content in the tail plasma sheet such that the cross-tail pressure gradients are directed toward the tail flanks. However, their simulation results of the region 1 current location (Karty et $a l$.) and the intensity ratio between region 1 and region 2 currents (Yang et al.) do not seem to be consistent with the observations.

Now, whether or not the hot plasma particles can be distributed in a torus configuration is an important issue. However, the main purpose of the present paper is not to solve this problem. In Appendix A we briefly discuss a possible scenario for the formation of HPT. Another problem is the maintenance of the HPT, because the poleward side of the HPT is unstable against the interchange instability (see, e.g., Wolf (1983)). In this regard, it is worthy of noting the results by $\mathrm{T}$. Yamamoto et al. (Interchange stability of the hot plasma torus in the terrestrial magnetosphere, 
submitted to Physics of Plasmas, 1996): The HPT can be maintained or impounded in the closed region; the impoundment is possible, on the nightside, due to the effect of reversal of the magnetic drift across the open-close boundary (when the IMF has a significant southward component), and it is also possible due to the outward pressure gradient in the low-latitude boundary layer (LLBL). When the condition for the HPT impoundment is violated, the interchange instability is fully developed, which can be regarded as a cause of the generation of auroral omega bands and torch-like structure (see Yamamoto et al. (1993, 1997a)). In the present paper, simply assuming the existence of the HPT, we will show that generation of the paired region 1 and region 2 FACs may be identified with polarization of the HPT distorted by the two-cell convection. From those theoretical works we may contend that the HPT really exists in the magnetosphere. Furthermore, there is observational evidence for its existence, but in the MLT range where the particles from the LLBL are detected: The Viking satellite observations of particle precipitation (Woch and Lundin, 1993; Woch et al., 1993) have shown that the poleward edge of the dayside plasma sheet lies inside a region of the particle population which may have its source in the LLBL. This means that the plasma sheet particles are concentrated in a region at latitudes lower than that of the open-close boundary because the LLBL is normally on closed field lines (Eastman and Hones, 1979). Then, the latitudinal profile of flux tube content of the plasma sheet particles must be peaked somewhere well inside the closed region, showing the presence of the HPT defined above, in the MLT sector of that Viking observation, i.e., dawn to prenoon sector.

\section{Simulation Model}

In this section we present a simulation model for the generation of the paired region 1 and region 2 field-aligned currents. For treating only the quasi-steady (time scale longer than several minutes) field-aligned currents we adopt the electrostatic model which neglects the effect of the Alfvén wave transmission in the magnetosphere. This approximation is valid because the Alfvén travel time to the ionosphere is less than several minutes. As is discussed by Yamamoto et al. (1996), inside the magnetically closed region (or the so-called slow-flow region (Wolf, 1983)) the divergence of the inertia current density is not responsible for quasi-steady production of a fieldaligned current. As is also studied by Yamamoto et al., we are allowed to consider only the hot plasma torus (HPT) with keV energy as the dominant source for the paired region $1 /$ region 2 FACs. Assuming that the (HPT) plasma pressure $(p)$ is isotropic and uniform along the field lines, we can show that the field-aligned current density $J_{\| \mathrm{i}}$ at the ionospheric height is given by (see Eq. (8) of Yamamoto et al.)

$$
J_{\| \mathrm{i}}=e \overline{\mathcal{V}}_{\mathrm{m}, \mathrm{i}} \cdot \nabla_{\mathrm{i}} \mathcal{E}
$$

where $e$ is the electronic charge and $\nabla_{\mathrm{i}}$ denotes the gradient on the ionospheric plane. Here $\mathcal{E}$ is the flux tube energy content, which is defined as the total kinetic energy of the plasma particles contained in a flux tube with unit cross-sectional area at the ionospheric height:

$$
\mathcal{E}=\int_{s_{\mathrm{i}}}^{s_{\mathrm{e}}} \frac{3 p}{2} \frac{B_{\mathrm{i}}}{B(s)} d s
$$

and $\overline{\mathcal{V}}_{\mathrm{m}, \mathrm{i}}$ is the averaged value of the ionospheric projection of the magnetic drift velocity per unit energy, which is defined as

$$
\overline{\mathcal{V}}_{\mathrm{m}, \mathrm{i}}=\frac{1}{R_{B}} \int_{s_{\mathrm{i}}}^{s_{\mathrm{e}}} \frac{V_{\mathrm{m}, \mathrm{i}}^{p}(s)}{W^{p}} \frac{1}{B(s)} d s \quad \text { and } \quad R_{B}=\int_{s_{\mathrm{i}}}^{s_{\mathrm{e}}} \frac{1}{B(s)} d s
$$

where $\boldsymbol{V}_{\mathrm{m}, \mathrm{i}}^{p}(s)$ is the ionospheric projection of the magnetic drift (gradient $B$ drift plus curvature drift) velocity, $\boldsymbol{V}_{\mathrm{m}}^{p}(s)$, of the proton fluid with the average energy $W^{p}, s$ is the field-aligned 
distance, $s_{\mathbf{e}}$ and $s_{\mathrm{i}}$ are at the equator and the ionospheric height; $B(s)$ and $B_{\mathrm{i}}$ are the magnetic field intensities at the distances $s$ and $s_{\mathrm{i}}$, respectively. The quantity $R_{B}$ represents the volume of a flux tube with one unit of magnetic flux.

In the present simulations the drift approximation can be used for the perpendicular motions of particles because the temporal and spatial scales of the large-scale field-aligned current are much greater than the ion cyclotron period and radius. As is discussed by Yamamoto et al. (1996), the inertia drift can be ignored. The drift velocity $\boldsymbol{V}_{\mathrm{m}}^{j}(s)$ is obtained by multiplying the magnetic drift velocity by the velocity distribution function of the $j$ species particles and integrating it over the velocity space.

For simple two-dimensional simulations, we make the following assumptions.

1. The latitudinal profile of the flux tube energy content $\mathcal{E}$ of the HPT at any local time has a single maximum.

2. The HPT is constituted by a singly ionized hydrogen plasma with keV energy. (Each particle species is treated as a fluid.)

3. The pressure of each particle species of the HPT is isotropic and uniform along the field lines; its average energy is constant in time and space.

4. The precipitation of the ions as well as the supply of ions from the ionosphere are ignored.

5. Injection of fresh particles into the closed region through the open-close boundary is ignored; the HPT particles exiting out of the closed region are removed from the simulation domain. (They are assumed to escape to the interplanetary space.)

6. The magnetic field lines are equipotential.

7. The ionospheric projection of a magnetic drift path is a circle with the center near the magnetic pole.

8. The average magnetic drift speed $\left(\bar{V}_{\mathrm{m}, \mathrm{i}}\right)$ projected onto the ionospheric plane (for each particle species) is constant.

9. The ionospheric (height-integrated) conductivities are uniform.

10. The ionospheric plane is perpendicular to the ambient magnetic field; the magnetic field strength is uniform there.

11. The solar wind convection is a priori given, in conformity with the Lyons (1985) model under the condition of IMF $B_{Z}<0$, i.e., southward interplanetary magnetic field.

Strictly speaking, owing to the assumptions (in 3-5) of constant average energy, no precipitation loss and no injection/ionospheric supply of the ions, the present simulations are applied only to the short-term ( $\$ 30 \mathrm{~min}$ ) behavior of the HPT deformation and associated FAC generation. In spite of this limitation, the present simulations will be shown to reproduce some remarkable features of the large-scale FACs observed during substorm periods.

In general, the conservation of particles of species $j$ (proton or electron) in a flux tube can be expressed in terms of two-dimensional variables defined on the ionospheric plane

$$
\frac{\partial}{\partial t} N^{j}+\operatorname{div}_{\mathrm{i}} \Gamma^{j}=Q^{j}-L^{j}
$$

where $\operatorname{div}_{\mathrm{i}}$ is operated on the ionospheric plane. In the above expression $N^{j}$ is the number of particles in a flux tube with unit (ionospheric) cross section:

$$
N^{j}=\int_{s_{\mathrm{i}}}^{s_{\mathrm{e}}} n^{j} \frac{B_{\mathrm{i}}}{B(s)} d s
$$

where $n^{j}$ is the number density of the species $j$; the particle influx $Q^{j}$ and outflux $L^{j}$ represent supply from and precipitation into the ionosphere, respectively; the lateral particle flux is defined as

$$
\boldsymbol{\Gamma}^{j}=\int_{s_{\mathrm{i}}}^{s_{\mathbf{e}}} n^{j}\left(\boldsymbol{V}_{\mathrm{E}, \mathrm{i}}(s)+\boldsymbol{V}_{\mathrm{m}, \mathrm{i}}^{j}(s)\right) \frac{B_{\mathrm{i}}}{B(s)} d s
$$


where $\boldsymbol{V}_{\mathrm{E}, \mathrm{i}}(s)$ and $\boldsymbol{V}_{\mathrm{m}, \mathrm{i}}^{j}(s)$ are the ionospheric projections of the electric and magnetic drift velocities, $\boldsymbol{V}_{\mathrm{E}}(s)$ and $\boldsymbol{V}_{\mathrm{m}}^{j}(s)$, of the $j$ species fluid. From assumption 3 the number density $n^{j}$ is found to be uniform along the field lines. Under assumption $6 \boldsymbol{V}_{\mathrm{E}, \mathrm{i}}(s)$ at any distance $s$ is identical to $\boldsymbol{V}_{\mathbf{E}}\left(s_{\mathrm{i}}\right)$, i.e., the electric drift velocity on the ionospheric plane. (Hereafter $\boldsymbol{V}_{\mathbf{E}}\left(s_{\mathrm{i}}\right)$ is simply denoted by $\boldsymbol{V}_{\mathrm{E}, \mathrm{i}}$.) The flux $\boldsymbol{\Gamma}^{j}$ in Eq. (4) is then written as

$$
\Gamma^{j}=N^{j}\left(V_{\mathrm{E}, \mathrm{i}}+\overline{\boldsymbol{V}}_{\mathrm{m}, \mathrm{i}}^{j}\right)
$$

where $\overline{\boldsymbol{V}}_{\mathrm{m}, \mathrm{i}}^{j}$ is the average magnetic drift velocity defined as

$$
\overline{\boldsymbol{V}}_{\mathrm{m}, \mathrm{i}}^{j}=\frac{1}{R_{B}} \int_{s_{\mathrm{i}}}^{s_{\mathrm{e}}} \boldsymbol{V}_{\mathrm{m}, \mathrm{i}}^{j}(s) \frac{1}{B(s)} d s .
$$

Note that the integration in Eq. (5) is equivalent to averaging over all the $j$ species particles in a magnetic flux tube volume when the density distribution is uniform along the field lines as assumed above. The particle conservation is now written as

$$
\frac{\partial N^{j}}{\partial t}+\operatorname{div}_{\mathrm{i}}\left\{N^{j}\left(\boldsymbol{V}_{\mathrm{E}, \mathrm{i}}+\overline{\boldsymbol{V}}_{\mathrm{m}, \mathrm{i}}^{j}\right)\right\}=Q^{j}-L^{j} .
$$

For the HPT protons both $Q^{p}$ and $L^{p}$ are assumed negligible (assumption 4 ). As will be addressed later, the total electron flux of $Q^{e}-L^{e}$ is considered to be given by $J_{\| \mathrm{i}} / e$.

On the basis of the particle conservation law Eq. (6), we suppose the population of "virtual particles" confined on the ionospheric plane as representing the cross-field dynamics of the HPT particles in the magnetosphere. The positions of individual virtual particles are given by the ionospheric projections of the HPT particles. Taking the projections of all the HPT particles, the "surface" number density of the virtual particles can be taken as $N^{j}$. In conformity with Eq. (6), we can assume that the virtual particles are moved by the electric drift due to the ionospheric field as well as by the magnetic drift of the velocities $\bar{V}_{\mathrm{m}, \mathrm{i}}^{j}$ defined in Eq. (5). In the actual simulations, taking the average energies of the HPT protons and electrons as $W^{p}=9 \mathrm{keV}$ and $W^{e}=3 \mathrm{keV}$ we specify the magnetic drift speeds as $\bar{V}_{\mathrm{m}, \mathrm{i}}^{p}=0.24 \mathrm{~km} / \mathrm{s}$ and $\bar{V}_{\mathrm{m}, \mathrm{i}}^{e}=0.08 \mathrm{~km} / \mathrm{s}$. This estimation is done for the dipole field line at $L=7 R_{\mathrm{E}}$ (see Fig. 9 of Yamamoto et al. (1996)).

Under assumption $3 J_{\| \mathrm{i}}$ in Eq. (1) can be expressed in terms of the surface density $N(=$ $N^{p} \cong N^{e}$ ) and the magnetic drift velocities $\overline{\boldsymbol{V}}_{\mathrm{m}, \mathrm{i}}^{j}$ of the virtual particles, that is,

$$
J_{\| \mathrm{i}}=e\left(\overline{\boldsymbol{V}}_{\mathrm{m}, \mathrm{i}}^{p}-\overline{\boldsymbol{V}}_{\mathrm{m}, \mathrm{i}}^{e}\right) \cdot \nabla_{\mathrm{i}} N \text {. }
$$

The above equation means that the field-aligned current density $J_{\| \mathrm{i}}$ can be determined by the production rate of space charges from the virtual particles (i.e., $J_{\| \mathrm{i}}=-\partial \sigma_{\mathrm{c}} / \partial t$ where $\sigma_{\mathrm{c}}$ is the surface charge density). This provides the actual way of evaluation of the FAC density $J_{\| i}$ in our simulations. In passing, it is worthy of noting that evaluation of the current density $J_{\| \mathrm{i}}$ does not need determination of the local plasma density $n$. This also implies that $J_{\| \mathrm{i}}$ is determined regardless of the density increase (or decrease) accompanying the plasma transport to inner (or outer) flux tubes of smaller (or larger) volumes.

On the basis of the thus obtained FAC density $J_{\| \text {i }}$, the electrostatic potential arising from the polarized HPT is calculated from the ionospheric current continuity

$$
J_{\| \mathrm{i}}=-\Sigma_{\mathrm{P}} \operatorname{div}_{\mathrm{i}} \boldsymbol{E}_{\mathrm{i}}
$$

where $\Sigma_{\mathrm{P}}$ is the height-integrated Pedersen conductivity and $\boldsymbol{E}_{\mathrm{i}}$ is the ionospheric electric field. Note that divergence of the Hall current is negligible for the uniform height-integrated conductivities (assumption 9). In the simulations $\Sigma_{\mathbf{P}}$ is taken as 8 mho. (This is the average level of $\Sigma_{\mathbf{P}}$ 


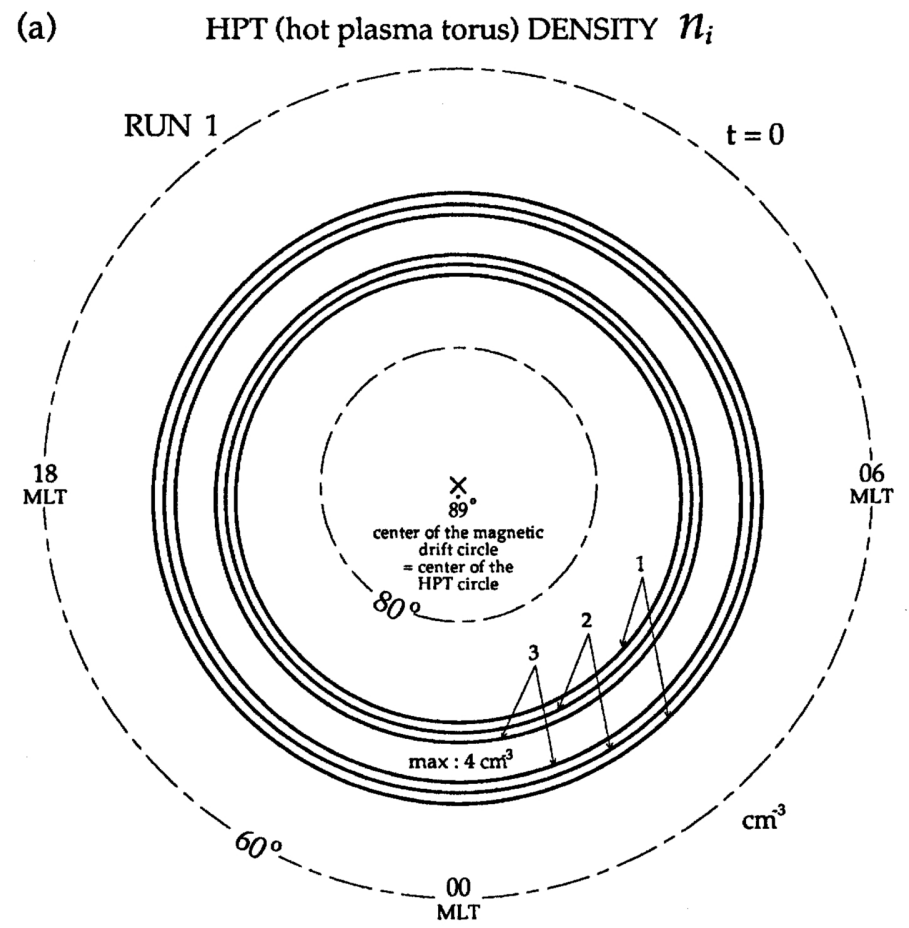

Fig. 1. Initial distribution of the HPT particles for run 1. They are distributed uniformly in the azimuthal direction. (a) Equicontours of the number density $n_{\mathrm{i}}$ are indicated, where $n_{\mathrm{i}}$ is proportional to the flux tube content $N$, as $n_{\mathrm{i}}=N /\left(R_{B} B_{\mathrm{i}}\right)_{0}$. In this paper the invariant latitude and MLT coordinates are used, and the magnetic pole is indicated by the cross. The center of the magnetic drift circle, assumed in our simulations, is indicated by the dot. This also gives the center of the circular isodensity contours. (b) Latitudinal profile $n_{\mathbf{i}}(x)$ and profile $n(x)$ of the corresponding HPT (local) density in the magnetosphere are indicated, in the upper panel, by the solid and dashed lines, respectively, where the origin of the latitudinal coordinate $x$ is set at the center of the HPT. In the lower panel, the ratio of $\left(R_{B} B_{\mathrm{i}}\right)(x) /\left(R_{B} B_{\mathrm{i}}\right)_{0}$ is plotted for the dipole field, where $\left(R_{B} B_{\mathrm{i}}\right)_{0}$ is the value of $R_{B} B_{\mathrm{i}}$ at $x=0\left(L=7 R_{\mathrm{E}}\right)$, i.e., $\left(R_{B} B_{\mathrm{i}}\right)_{0}=1.32 \times 10^{7} \mathrm{~km}$. The local density $n$ is related to $n_{\mathrm{i}}$, as $n(x)=\left(\left(R_{B} B_{\mathrm{i}}\right)_{0} /\left(R_{B} B_{\mathrm{i}}\right)(x)\right) n_{\mathrm{i}}(x)$. (c) Distribution of the HPT particles with an azimuthal density gradient toward local midnight. This is used for obtaining the initial HPT distribution for run 4 (see text).

in the nightside auroral zone, as has been shown by Hardy et al. (1991, Fig. 2).) The ionospheric potential is solved under the boundary condition that the potential vanishes at a latitude of $62^{\circ}$. Note that the ionospheric potential distribution is essentially the same as the magnetospheric potential distribution, under the assumption of equipotential field lines. The $E \times B$ drift velocity of the virtual particles is calculated assuming that the magnetic field strength on the ionosphere is $6 \times 10^{4} \mathrm{nT}$.

In our model the trajectory of a magnetically drifting virtual particle (without electric drift) is assumed to be a circle (assumption 7). As is noted in the introduction, this is a good approximation to the ionospheric projection of the magnetic drift path in the closed region when the coordinates of invariant latitude and MLT are used. Accordingly, our simulation is implicitly based on the invariant latitude and MLT coordinates. Taking into account the satellite observations of the energetic electron intensity by McDiarmid et al. (1975), the center of the circle representing a magnetic drift shell projected onto the ionosphere is set at $89^{\circ}$ latitude on the midnight meridian (see Fig. 1(a)). The HPT configuration in the absence of $E \times B$ convection 

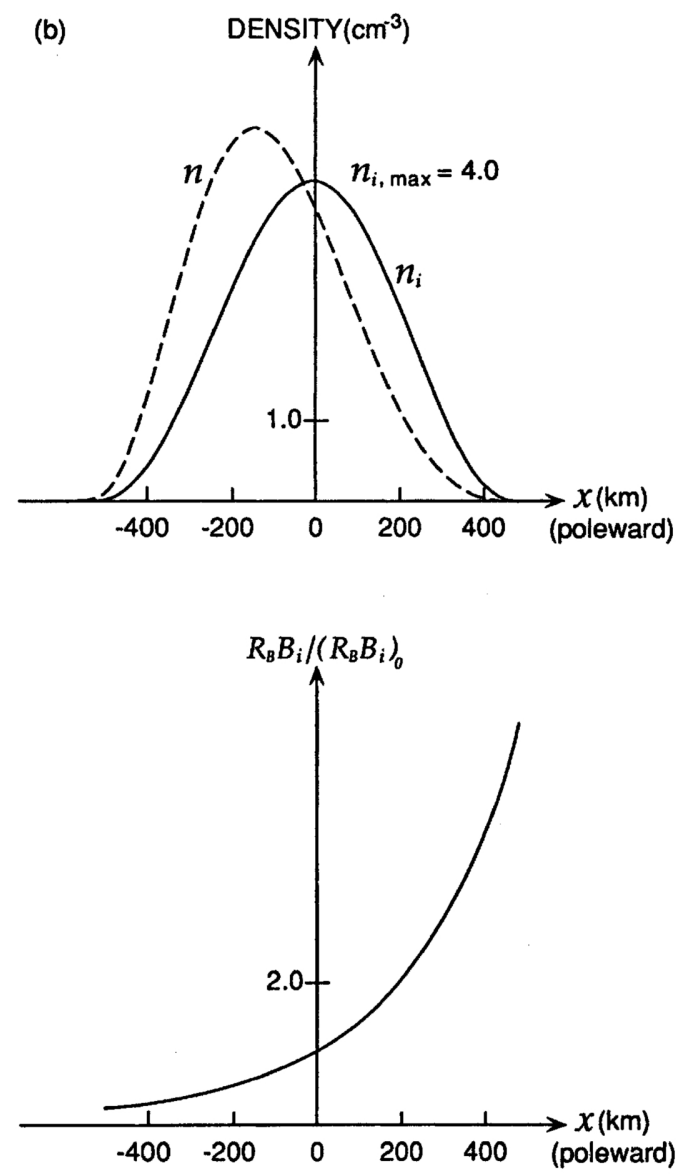

Fig. 1. (continued).

should be identical to the magnetic drift shells, namely, the ionospheric projection of the HPT is an annulus encompassed with two concentric circles. In our paper such a state of the HPT is called the ground state. The ground state is assumed as the initial condition of the HPT particles for simulation runs 1-3: in Fig. 1(a) the initial distribution of the HPT virtual particles is shown by the isodensity contours. Here indicated is the number density $n_{\mathrm{i}}$ of the virtual particles, which is defined as $n_{\mathrm{i}}=N /\left(R_{B} B_{\mathrm{i}}\right)_{0}$ where $\left(R_{B} B_{\mathrm{i}}\right)_{0}$ is the value of $R_{B} B_{\mathrm{i}}$ at the center line of the (initial) HPT belt, and the variation of $R_{B} B_{\mathrm{i}}$ along the magnetic drift path is neglected (see Section 2 of Yamamoto et al. (1996)). Actually, $\left(R_{B} B_{\mathrm{i}}\right)_{0}$ is taken as $1.32 \times 10^{7} \mathrm{~km}$, which is the value of $R_{B} B_{\mathrm{i}}$ for the dipole field line at $L=7 R_{\mathrm{E}}$. Note that the density $n_{\mathrm{i}}$ is proportional to the flux tube content ("surface" density) $N$ defined in Eq. (3), while $n_{\mathrm{i}}$, only at the initial HPT center line, is equal to the HPT (local) density in the magnetosphere. In Fig. 1(b) (upper panel) the latitudinal profile $n_{\mathrm{i}}(x)$ of the virtual particles is shown by the solid line, where the $x$ direction is perpendicular to the HPT center line and the HPT width $\left(2 L_{x}\right)$ is taken as $2 L_{x} \sim 950$ $\mathrm{km}\left(\sim 8.7^{\circ}\right.$ in latitude). (Note that the HPT is defined as the plasma concentration in terms of the flux tube content $N$.) For reference, in Fig. 1(b), the dashed line shows the profile $n(x)$ of the corresponding HPT (local) density in the magnetosphere: The density $n(x)$ is given by $n(x)=\left(\left(R_{B} B_{\mathrm{i}}\right)_{0} /\left(R_{B} B_{\mathrm{i}}\right)(x)\right) n_{\mathrm{i}}(x)$, and the factor $\left(R_{B} B_{\mathrm{i}}\right)(x)$ increases with latitude and actu- 
(c)

HPT DENSITY $n_{i}$

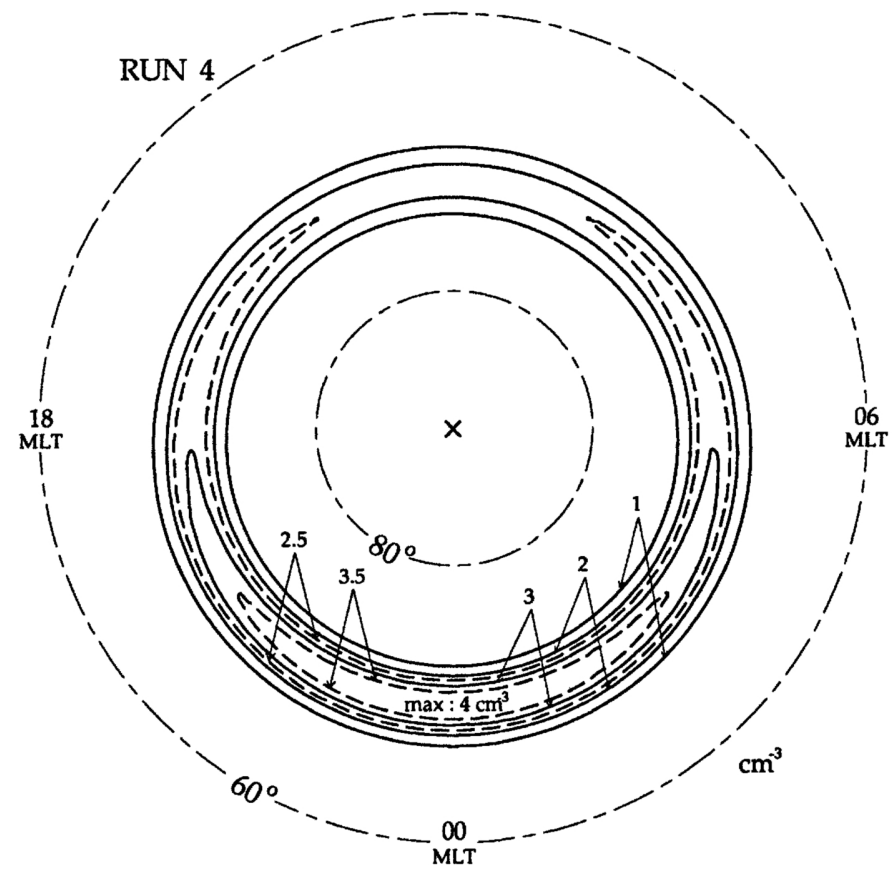

Fig. 1. (continued).

ally it is evaluated by using the Earth dipole field (see lower panel), where it is assumed that the dipole magnetic shell at $L=7 R_{\mathrm{E}}$ has a foot point at the initial position of the HPT center line. Figure 1(c) shows the HPT distribution $n_{\mathrm{i}}$ with a density gradient (toward local midnight) in the azimuthal $(y)$ direction, which is used in run 4 to examine the effect of the (enhanced) azimuthal pressure gradient on the field-aligned current generation. (In this paper the azimuthal direction is defined to be the direction of the average magnetic drift velocity $\overline{\boldsymbol{V}}_{\mathrm{m}, \mathrm{i}}^{j}$. ) The latitudinal profile $n_{\mathrm{i}}(x)$ of the HPT density in Fig. 1(c) is made similar to that shown in Fig. 1(b).

When the HPT is distorted, polarization occurs due to oppositely directed magnetic drifts of the HPT electrons and protons. The generated space charges are thought to be drained primarily by electrons moving quickly along the field lines. (Negative space charges will be carried away by the HPT electrons precipitating to the ionosphere, while positive ones will be neutralized by upflowing electrons of ionospheric origin.) As a first approximation, we ignore the precipitation of ions as well as the supply of ions from the ionosphere (assumption 4). In fact, the DMSP satellites have shown that the electron precipitation flux is usually 5 to $10^{3}$ times larger than the ion precipitation flux. We are then allowed to follow only the dynamics of the HPT protons as representing the whole HPT dynamics in a plane perpendicular to the magnetic field. Injection of fresh particles into the closed region through the open-close boundary including the cusp/LLBL regions is neglected (assumption 5), whereas the HPT particles exiting out of the closed region are assumed to escape to the interplanetary space, namely, they are actually removed from the simulation domain. Here the magnetically open region is defined to be the region encircled by the poleward boundary of the HPT initially given. (Considering the fact that a particle leaving the closed region takes some time to escape away from the terrestrial region, the latitudinal profile of $n_{\mathrm{i}}$ just outside the (dayside) closed region is made to decrease gradually with the distance from 
the open/close boundary; the characteristic distance (in latitude) for the decrease of $n_{\mathrm{i}}$ is assumed to be of the order of $100 \mathrm{~km}$ (see Appendix A).) In the simulations, as will be shown later, the particle loss occurs only in the dayside region centered around local noon. (The particle injection from the LLBL and cusp regions might play an important role in generation of the FACs therein, which is, however, beyond the scope of the present study.)

The solar wind convection pattern is specified as shown in Fig. 2(a), where the equipotential contours are indicated. Note that the electric drift velocity is tangent to the equipotential contours. This potential distribution is produced from the space charges (Fig. 2(b)) given in the open region encircled by the poleward boundary of the HPT. In conformity with the space charge distributions in the polar cap theoretically predicted (for IMF $B_{Z}<0$ and $\left|B_{Y} / B_{Z}\right|<1$ ) by Lyons (1985), negative space charges are distributed over the eveningside semicircular domain with increasing density toward the open-close boundary in the premidnight sector while the positive charge distribution is the inverse of the negative charge distribution with respect to the noon-midnight meridian. The space charge density is determined so that the resulting potential drop across the open region is several tens of kilovolts which may be a reasonable level during the moderately disturbed condition. Also, the maximum speed of the resulting $E \times B$ convection in the closed region is $0.25 \mathrm{~km} / \mathrm{s}$.

In the first simulation (run 1), the solar wind convection in Fig. 2 is persistently impressed upon the HPT in the magnetosphere. The purpose of run 1 is to see how the HPT is deformed purely by the solar wind convection and estimate the electric potential as well as the field-aligned currents resulting from this HPT deformation. In the calculation of the HPT deformation we do not include the electric fields which can arise from the polarization of the HPT.

The second simulation (run 2) is the continuation of run 1, but it includes the electric fields self-consistently produced from the polarization of the HPT. (The specifications of runs 1-4 are briefly summarized as a diagram in Fig. 3.) The "initial" configuration of the HPT in run 2 is given by the deformed HPT which is finally obtained in run 1, being responsible for the generation of FACs consistent with the observations. In run 2 we further follow the HPT deformation in the presence of both the solar wind convection and the convection field from the HPT polarization. (Hereafter the former is referred to as the external-convection (field) and the latter is the internalconvection (field).) Specifically, in run 2, we make an attempt to simulate a possible situation that the potential drop across the open region is controlled by the solar wind when the IMF $B_{Z}$ is negative, which implies that the solar wind acts as a voltage generator. (As is discussed by Yamamoto et al. (1996), this assumption is supported by the observed relationship between the FAC intensities and the ionospheric conductivities which was obtained by Fujii and Iijima (1987).) In the actual calculation the polar cap potential drop of the external-field is adjusted at each time step so that the potential drops of the external- and internal-fields add up to the external-potential drop $(40.6 \mathrm{kV})$ initially given.

Run 3 is also the continuation of run 1 , but the solar wind convection is switched off while the internal-field is included. The initial configuration of the HPT in run 3 is again given by the deformed HPT finally obtained in run 1 . The purpose of run 3 is to examine whether or not the positive feedback is possible in the relationship between the HPT deformation and the internalfield generation, namely, the FACs can be intensified, through the HPT deformation/polarization, only by the internal-field.

Run 4 is executed to show how the distributions of the FACs and the internal-field are modified by enhancement of the HPT density (azimuthal) gradient toward local midnight. Except for the midnight-directed density gradient, run 4 is the same as run 3 ; it includes only the internal-field, and its initial HPT configuration is obtained by impressing the solar wind convection (Fig. 2(a)) upon the HPT with an azimuthal density gradient (Fig. 1(c)) for the same period as in run 1. (As will be seen from the results of runs 1-3 where the initial HPT is azimuthally uniform, the HPT distortion is accompanied by the occurrence of azimuthal density gradients 
(a) EXTERNAL-POTENTIAL (solar wind convection)

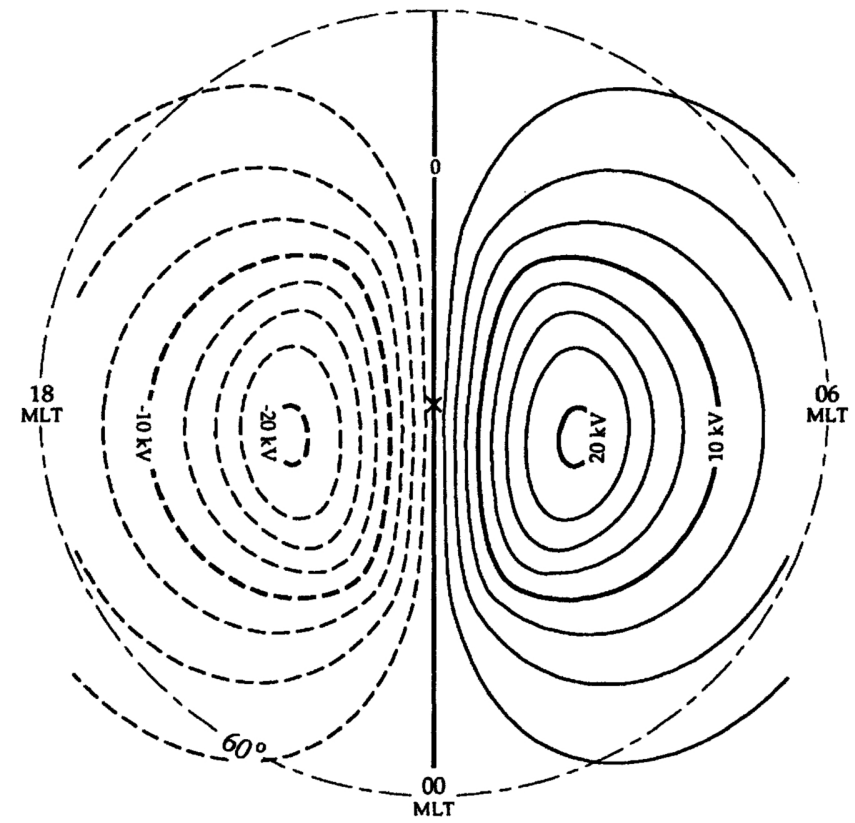

(b) SPACE CHARGE DENSITY (solar wind convection)

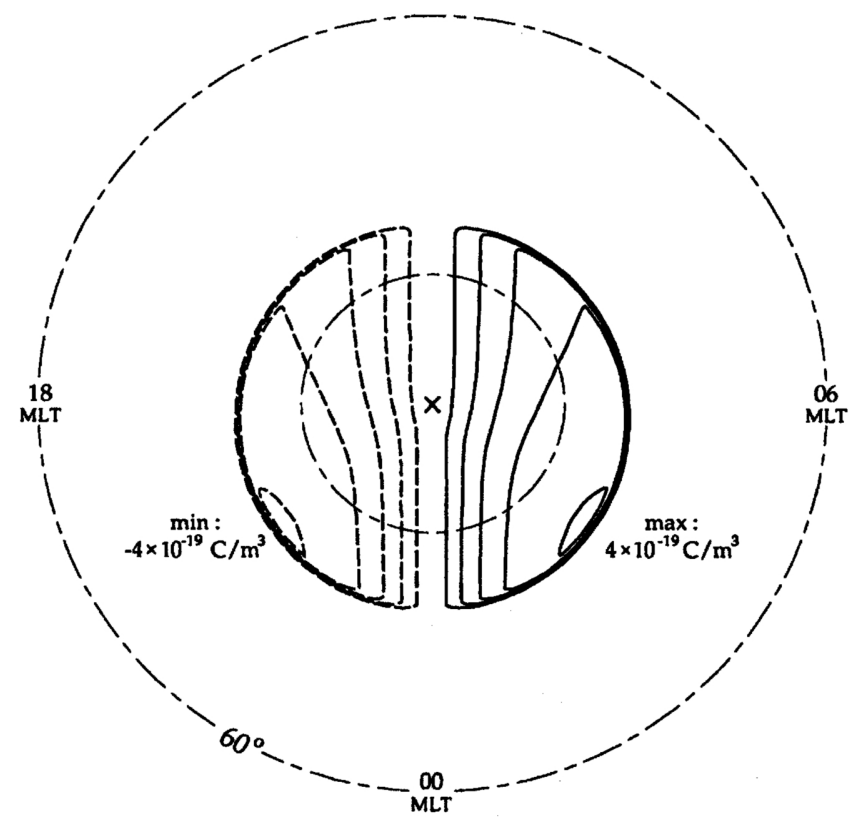

Fig. 2. (a) Equipotential contours for the solar wind convection (external-potential) assumed in the simulations. Solid and dashed contours are for positive and negative potentials, respectively. The contour interval is $2.5 \mathrm{kV}$. The potential distribution is obtained from the space charge distribution in (b), by numerically solving the Poisson's equation under the boundary condition that the potential vanishes at a latitude of $50^{\circ}$. (b) Space charge density responsible for the solar wind convection. The maximum density of $4 \times 10^{-19} \mathrm{C} / \mathrm{m}^{3}$ corresponds to a field-aligned current density of $0.09 \mu \mathrm{A} / \mathrm{m}^{2}$ when the height-integrated Pedersen conductivity in the polar cap is taken as 2 mho. Solid and dashed contours are for positive and negative charge densities, respectively, and the contour interval is $7.8 \times 10^{-20} \mathrm{C} / \mathrm{m}^{3}$. 

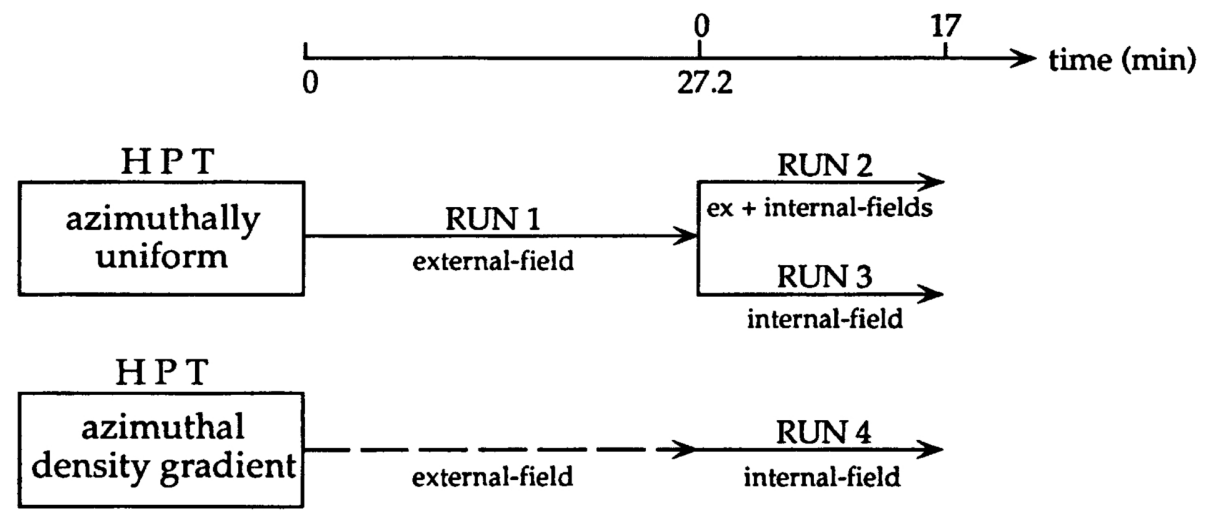

Fig. 3. Diagram for main specifications of simulation runs 1-4.

(which are toward midnight in the region 2 zone). Particularly, in run 4, the midnight-directed density gradient is added to the initial HPT which is used for runs 1-3.)

The numerical simulations are performed in the (ionospheric) polar region at invariant latitudes greater than $62^{\circ}$, by using a particle-in-cell code (e.g., Okuda, 1985). This circular simulation domain is covered with $128 \times 512$ grid meshes in polar coordinate $(r, \phi)$. The magnetic local time increases in the counterclockwise direction and the midnight meridian is set at the radial axis pointing downward. The grid spacing $\Delta r$ is then about $24 \mathrm{~km}$, and $r \Delta \phi$ is, for example, about $27 \mathrm{~km}$ at an invariant latitude of $70^{\circ}$. In the two-dimensional guiding center model the grid spacing is not required to be less than the ion Larmor radius nor the Debye length, and the time step is not required to be less than the ion cyclotron period. The guiding center positions of particles are advanced by using the predictor-corrector method (Lee and Okuda, 1978). The time step $\Delta t$ is taken small enough to satisfy the Courant condition of $V_{\max } \Delta t<\Delta r$ (or $r \Delta \phi$ ), where $V_{\max }$ is the maximum particle speed. The results to be presented are from runs using a time step of $\Delta t=20.4 \mathrm{~s}$ for run 1 and $10.2 \mathrm{~s}$ for runs $2-4$. We have confirmed that the simulation results are not appreciably changed by halving the time step. The HPT proton population (virtual particles) consists of 90,400 superparticles. To create a nonuniform density, they have different weights in charge $q$ and mass $M$, but the ratio of $M / q$ is fixed. There is no need for loading the background cold particles (in the magnetosphere), because the charge separation as is responsible for generation of quasi-steady large-scale field-aligned currents does not take place in this particle population. (For detailed discussion, see Section 1 of Yamamoto et al. (1996).) In runs 2-4, electrostatic fluctuations with wavelengths of, typically, a few hundred kilometers could be amplified by the interchange instability. (According to Yamamoto et al. (1993, 1996), this interchange instability can occur on the high-latitude side of the HPT due to the oppositely directed magnetic drifts of the HPT protons and electrons.) To focus the present analysis on the global generation of field-aligned currents, we suppress the growth of these fluctuations by smoothing the azimuthal distributions of particles and electric fields within the range of such wavelengths. In other words, we implicitly assume that the HPT is impounded in the closed region by the effects of magnetic drift reversal across the open-close boundary and/or the outward pressure gradient in the LLBL, as is addressed in the introduction. 


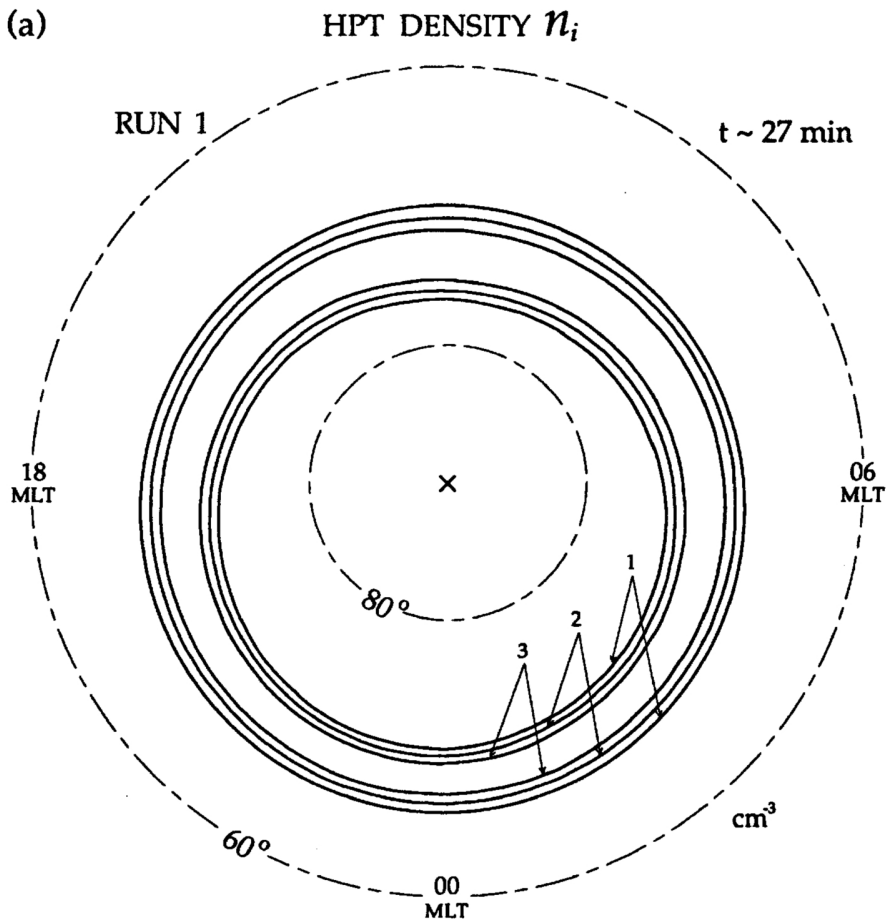

Fig. 4. Results of run 1 at the time of $\sim 27 \mathrm{~min}$. (a) Number density $n_{\mathrm{i}}$ of the deformed HPT. (b) Magnetospheric density $n$ of the HPT in (a). The two-dimensional pattern (upper) and the latitudinal profile $n(x)$ along the midnight meridian (lower) are shown. The corresponding profile of $n_{\mathrm{i}}(x)$ is also indicated by the dashed line. The origin of the latitudinal coordinate $x$ is set at the center of the undistorted (initial) HPT (Figs. 1(a) and $1(\mathrm{~b}))$. (c) Field-aligned current density resulting from the HPT deformation. Dashed equicontours are for upward FAC density $J_{\| \mathrm{i}}(>0)$ at the ionospheric height and solid contours are for downward FAC density $J_{\| \mathrm{i}}(<0)$. For the region 1 FACs, the contours begin at $\pm 0.2 \mu \mathrm{A} / \mathrm{m}^{2}$ and are incremented thereafter by \pm 0.4 $\mu \mathrm{A} / \mathrm{m}^{2}$. For the region $2 \mathrm{FACs}$, the contour interval is $0.2 \mu \mathrm{A} / \mathrm{m}^{2}$. (d) Electric potential (internal-potential) distribution corresponding to the FAC distribution in (c). Solid and dashed contours are for positive and negative potentials, respectively, and the contour interval is $2 \mathrm{kV}$.

\section{Simulation Results}

\subsection{Run 1}

The simulation results of run 1 are presented in Figs. 4 and 5. Figure 4(a) shows the number density $n_{\mathrm{i}}$ of the HPT virtual particles, which is obtained after impressing the solar wind convection for about $27 \mathrm{~min}$. (Recall that the initial HPT distribution in this run is shown in Fig. 1(a).) Figure 4(b) shows the corresponding plasma density $n$ in the magnetosphere, which is calculated from the relation of $n=\left(R_{B} B_{\mathrm{i}}\right)_{0} /\left(R_{B} B_{\mathrm{i}}\right) n_{\mathrm{i}}$ : the two-dimensional pattern is shown in the upper panel and the latitudinal profile along the midnight meridian is in the lower panel. The meaning of this density distribution is discussed in Appendix B. Figure 4(c) shows the fieldaligned current density, estimated at the ionospheric height, resulting from the HPT deformation in Fig. 4(a). Figure 4(d) shows the corresponding internal-potential arising from the polarized HPT at the time $t$ of $\sim 27 \mathrm{~min}$. This is calculated from the FAC distribution in Fig. 4(c), on the basis of the Pedersen current closure assumed in the preceding section. Figures 5(a) and 5(b) show the temporal variations of the maximum (region 1) FAC density and the (internal-) potential drop across the polar cap, respectively. Here the maximum FAC density is evaluated as an average of 

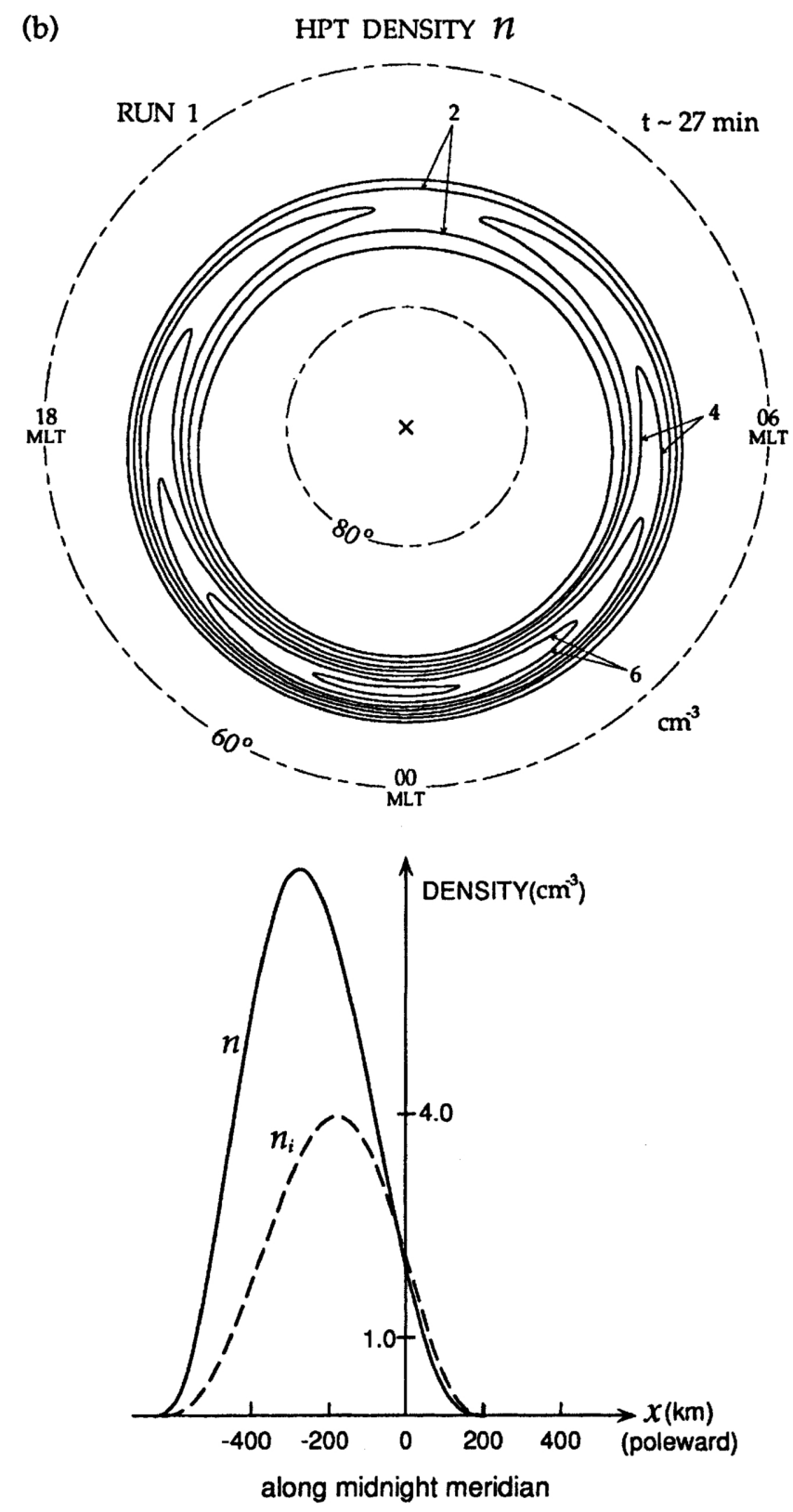

Fig. 4. (continued).

two peak magnitudes of the region 1 FAC densities in the dawn and dusk sectors.

From Fig. 4(c) it is found that the FAC pattern in this simulation is consistent with the observed pattern of the paired region 1 and region 2 FACs (Iijima and Potemra, 1976; 1978). The field-aligned currents are not generated in the MLT sectors where the HPT center line is parallel to the direction of the particle magnetic drift. (The FAC generation in the midnight sector will be discussed in Yamamoto et al. (submitted, 1996).) From the symmetry of the solar 
(c) FIELD-ALIGNED CURRENT DENSITY (magnetic drift)

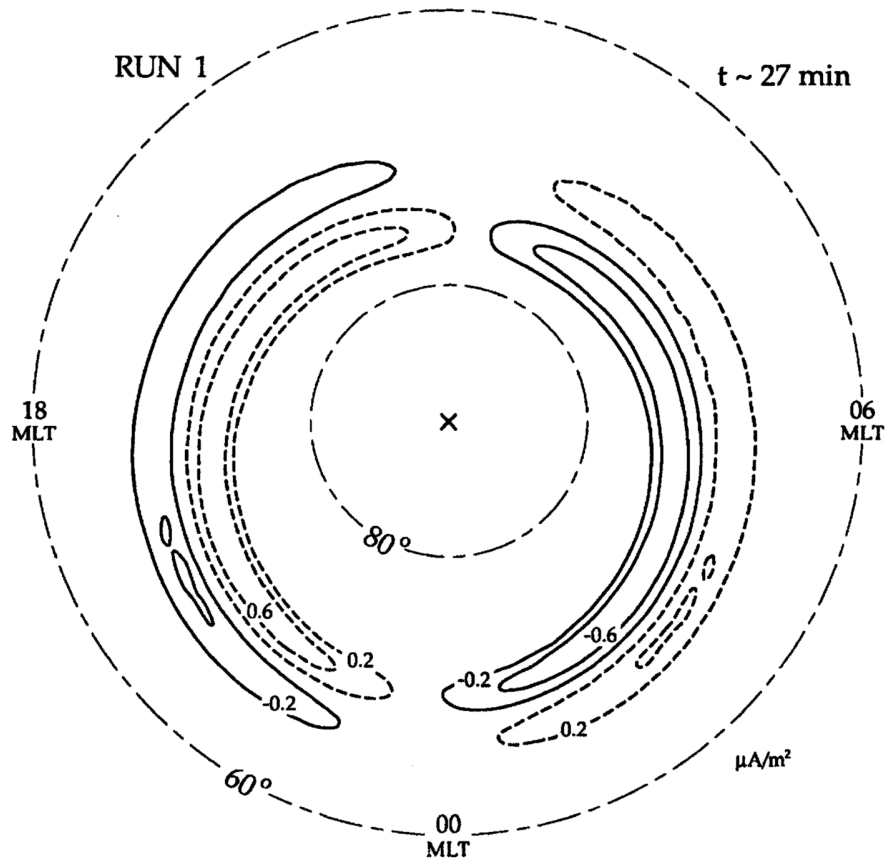

(d) INTERNAL-POTENTIAL (HPT polarization)

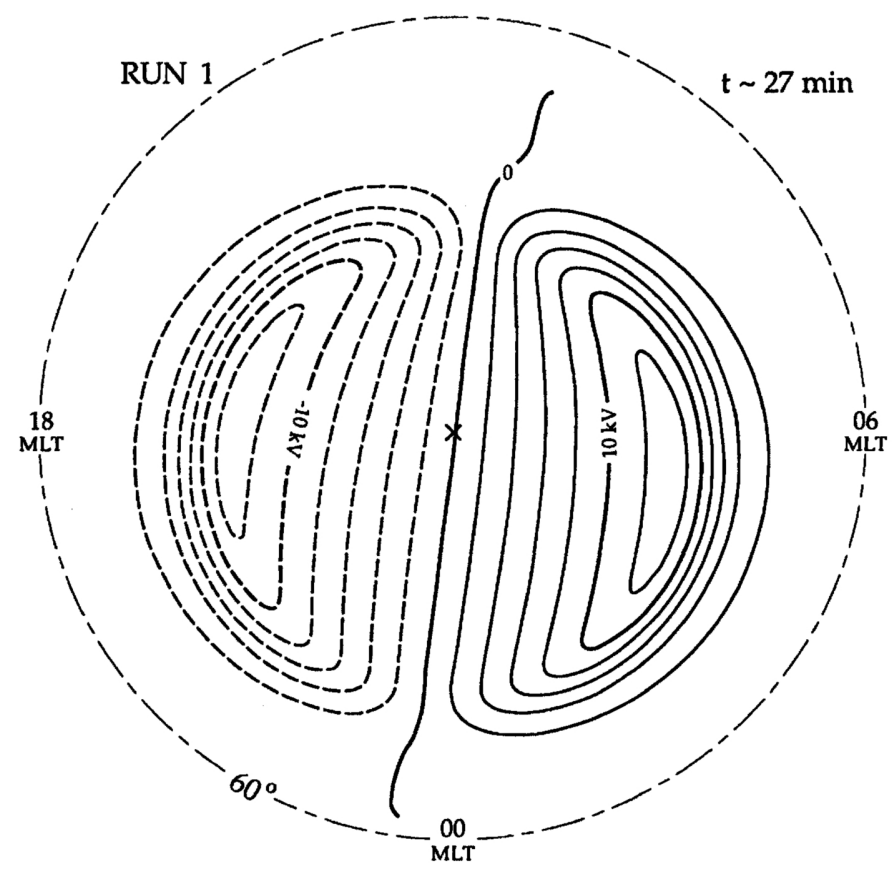

Fig. 4. (continued). 


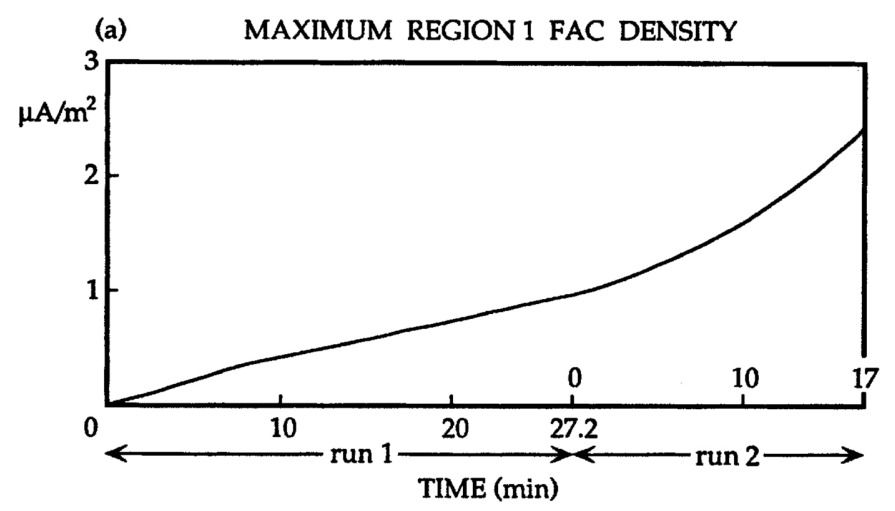

(b) POLAR CAP POTENTIAL DROP OF INTERNAL-FIELD

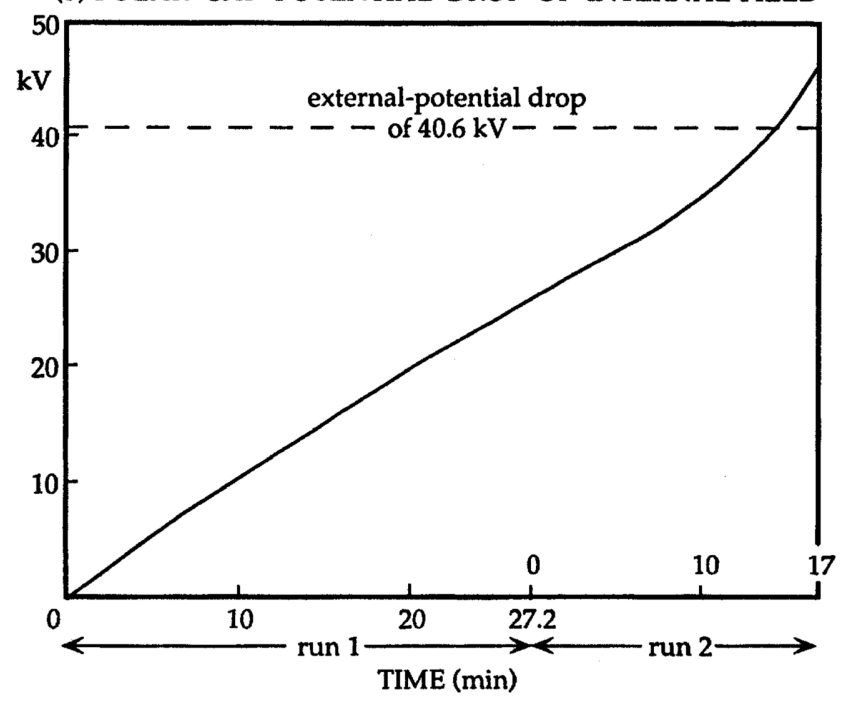

Fig. 5. Temporal variations of (a) the maximum (region 1) FAC density and (b) the (internal-) potential drop across the polar cap, in the consecutive simulation runs 1 and 2.

wind convection with respect to the noon-midnight meridian, the regions of vanishing FACs are expected to be positioned at local midnight and noon. However, due to the westward magnetic drift of the HPT protons, such regions shift from local midnight and noon, by the longitudinal distance of $\lesssim \bar{V}_{\mathrm{m}, \mathrm{i}}^{p} \cdot t \sim 0.24 \mathrm{~km} / \mathrm{s} \cdot 1600 \mathrm{~s} \sim 390 \mathrm{~km}$ on the ionospheric plane. This estimation is consistent with the FAC pattern in Fig. 4(c). In this simulation the region 1 FAC intensity is greater than the region 2 FAC intensity. This is explained as follows. Basically, nearer to local midnight (or noon) the HPT is displaced to lower (or higher) latitudes by the greater latitudinal (antisunward) component of the solar wind convection flow. Such distortion further proceeds in the higher-latitude portion of the HPT, due to the greater latitudinal flow. Namely, the angle $\theta$ between the equicontours of the density $n_{\mathrm{i}}$ and the magnetic drift direction of $\bar{V}_{\mathrm{m}, \mathrm{i}}^{j}$ is greater at higher latitudes. (The angle $\theta$ is called the HPT distortion angle, representing the degree of distortion.) For this reason, the region 1 FAC has greater intensities than the region 2 FAC.

As a summary of the results of run 1 , it is stated that the solar wind convection with a polar cap potential drop of several tens of kilovolts can sufficiently deform the HPT, during a 
relatively short period of $\lesssim 30 \mathrm{~min}$, to produce the paired region $1 /$ region 2 FACs with current densities of $\sim 1 \mu \mathrm{A} / \mathrm{m}^{2}$. As will be shown in the subsequent simulation runs, the FAC generation can be promoted by the simultaneous development of the (internal-) electric field arising from the HPT polarization. This is because as is shown in Fig. 4(d) the internal-convection is similar to the solar wind convection in the structure of twin vortex cells with antisunward flows in the polar cap, and the internal-convection is shortly strengthened to be comparable to the externally imposed solar wind convection (see Figs. 4(d) and 5(b)).

\subsection{Run 2}

The simulation results of run 2 are presented in Figs. 5 and 6. Figures 5(a) and 5(b) show the temporal variations of the maximum (region 1) FAC density and the internal-potential drop across the polar cap, respectively. Note that they change continuously from the maximum FAC density and the potential drop at $t \sim 27 \mathrm{~min}$ in run 1 . Figure $6(\mathrm{a})$ shows the density $\left(n_{\mathrm{i}}\right)$ profiles of the HPT at the times of about $7 \mathrm{~min}$ and $14 \mathrm{~min}$. Here indicated is the time elapsed after we start run 2 with the HPT distribution finally obtained in run 1. Figure 6(b) shows the fieldaligned current densities resulting from the HPT deformations in Fig. 6(a). Figure 6(c) shows the superposition of the internal- and external-potentials at these times.

Run 2 treats the more realistic case than run 1: the addition of the internal-potential drop and the external-potential drop is fixed at that $(40.6 \mathrm{kV})$ of the external-potential imposed in run 1 , assuming that the solar wind acts as a voltage generator in the interaction with the open field lines. (Note that the potential drop of the superposition of the internal and external fields is not fixed, but as a result kept at about $40 \mathrm{kV}$ (see Fig. 6(c)). The external-potential with reversed polarity is imposed during the later period of run 2 when the internal-potential drop exceeds 40.6 $\mathrm{kV}$.) In contrast with the external-(solar wind) convection in Fig. 2(a), the "total" convection in Fig. 6(c), more than $60 \%$ of which is of internal origin (see Fig. 5(b)), has well-defined throats inside the region of the HPT, where the throat is defined as the region in which the antisunward convection flow bifurcates into the westward and eastward flows in the midnight sector or vice versa in the noon sector. For this reason, the HPT displacement to lower (or higher) latitudes is enhanced particularly in the limited MLT sector centered at the midnight (or noon) throat. (On the dayside, the HPT distribution is limited within the closed region because a plasma leaving the closed region is assumed to escape, along the open field lines, from the terrestrial region. Before long, due to this boundary condition, the HPT has a steep density gradient normal to the open-close boundary around 11 MLT, which is evident in the density profile at $t \sim 14$ min in Fig. 6(a).) Figure 7 shows how the HPT is displaced from its initial location; the initial (undistorted) HPT is superposed on the distorted HPT at $t \sim 14 \mathrm{~min}$ in run 2, and the arrows indicate the magnetic drift directions which are tangent to the isodensity $\left(n_{\mathrm{i}}\right)$ contours of the initial HPT. In this figure the above-described distortion on the nightside is more pronounced on the high-latitude boundary of the HPT than on the low-latitude boundary. This is explained by the fact that the internal-convection flow turns to the longitudinal direction around the interface between the region 1 and region 2 FACs. Thus a pair of the region 1 FACs, generated on the high-latitude side of the HPT, are enhanced particularly in the premidnight and postmidnight sectors. Notably, this process may correspond to the disruption of the tail current (e.g., Nagai, 1982). In this context the tail current is assumed to be carried by the particles in the high-latitude portion of the HPT, which is regarded as the boundary plasma sheet (BPS). In Fig. 7, a magnetic drift path inside the BPS at the initiation of run 2 is indicated by the thick arc. At $t=0$, the tail current flows along this circular path. At the later times, say at $t \sim 14 \mathrm{~min}$, the HPT particles (i.e., carriers of the tail current) evacuate the initial location of the BPS, which means that the tail current completely disrupts there. Note that the region of peaked region 1 (downward) FAC on the dawnside is geometrically connected to that of peaked region 1 (upward) FAC on the duskside by a magnetic drift path, namely, a disrupted current path. From the potential pattern 
(a)

HPT DENSITY $n_{i}$

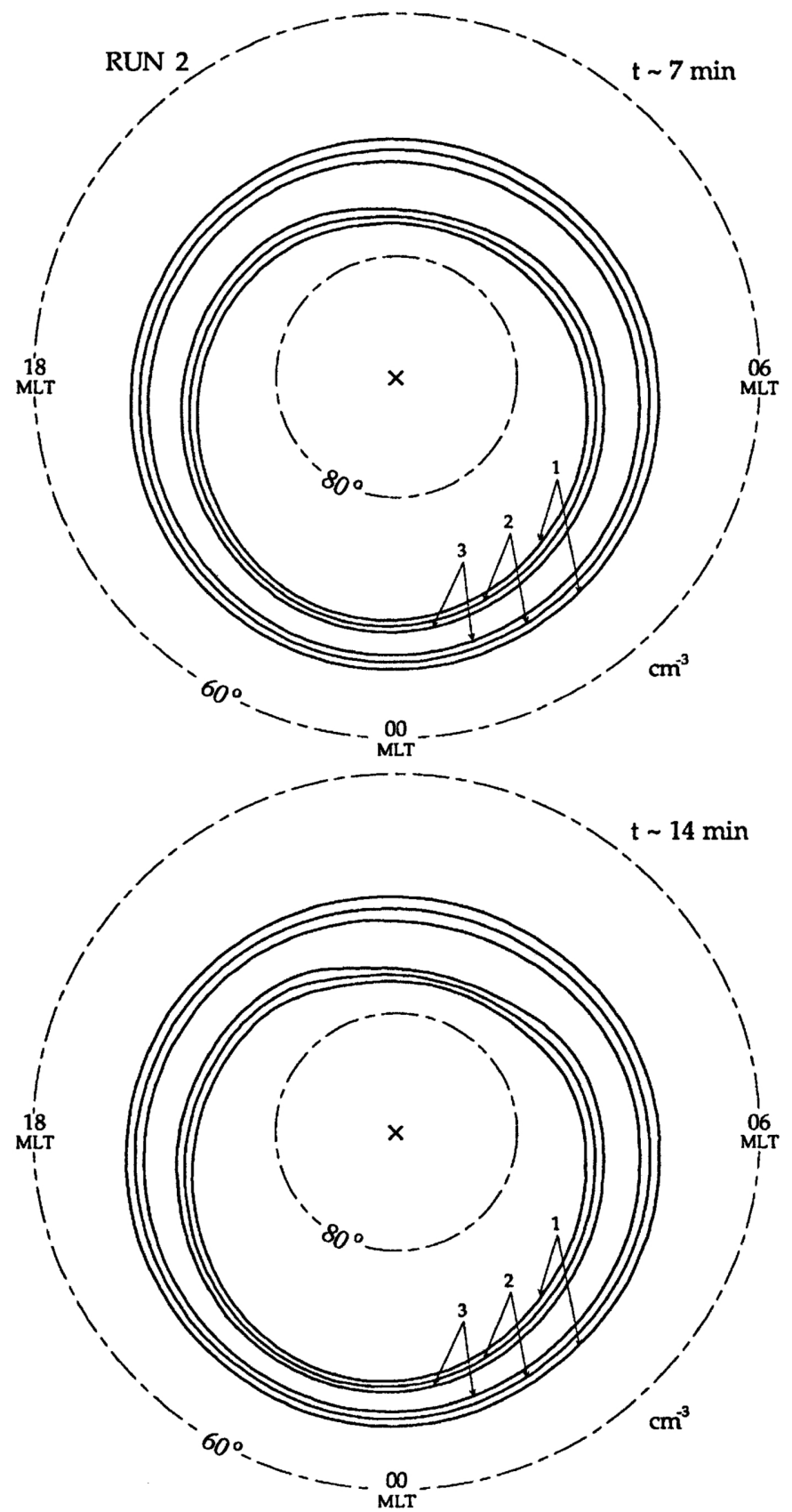

Fig. 6. Results of run 2 at $t \sim 7$ min and $t \sim 14$ min. Figures 6(a) and 6(b) are in the same format as Figs. 4(a) and $4(\mathrm{c})$. Figure $6(\mathrm{c})$ shows the superposition of internal- and external-potentials, where the contour interval is $2.5 \mathrm{kV}$. 
(b) FIELD-ALIGNED CURRENT DENSITY (magnetic drift)

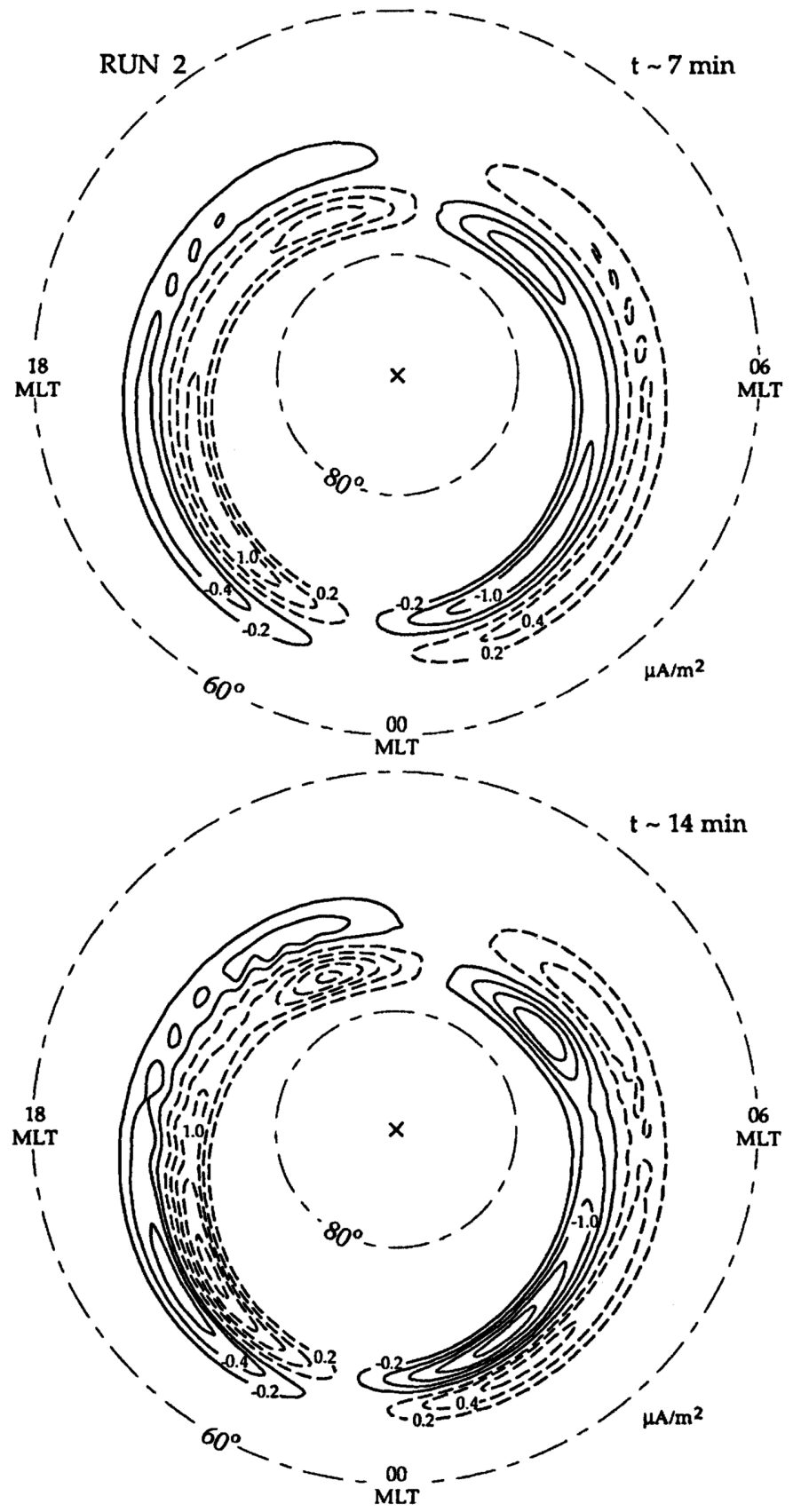

Fig. 6. (continued). 
(c) EXTERNAL+INTERNAL-POTENTIALS

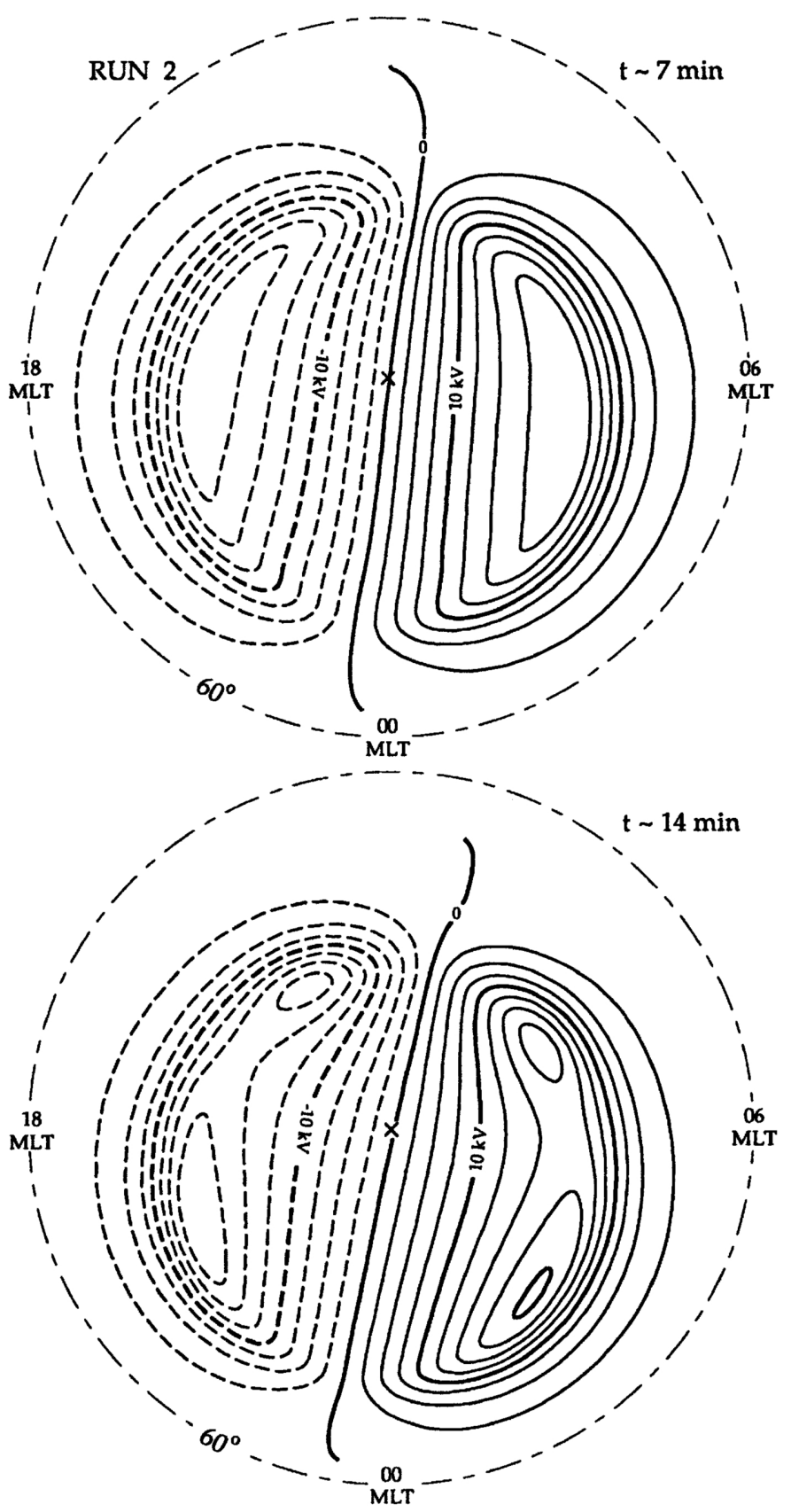

Fig. 6. (continued). 


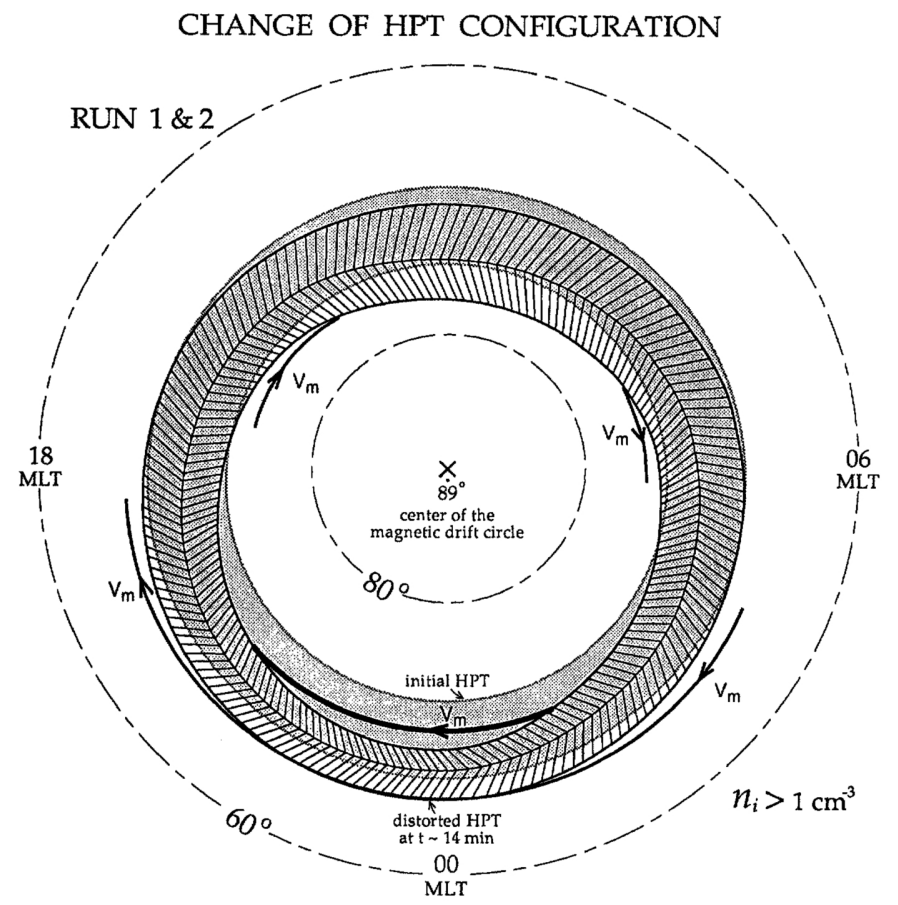

Fig. 7. A change of the HPT configuration in the course of runs 1 and 2. The shaded region shows the initial (undistorted) HPT with $n_{\mathrm{i}}>1 \mathrm{~cm}^{-3}$, and the lined region shows its distorted distribution at $t \sim 14 \mathrm{~min}$ in run 2. The radial density $\left(n_{\mathrm{i}}\right)$ gradient has opposite directions in two zones of different lined patterns, in each of which the region 1 or region 2 FAC is developed. The undistorted HPT is uniform along the magnetic drift shells, which are represented by concentric circles with the center at the indicated position.

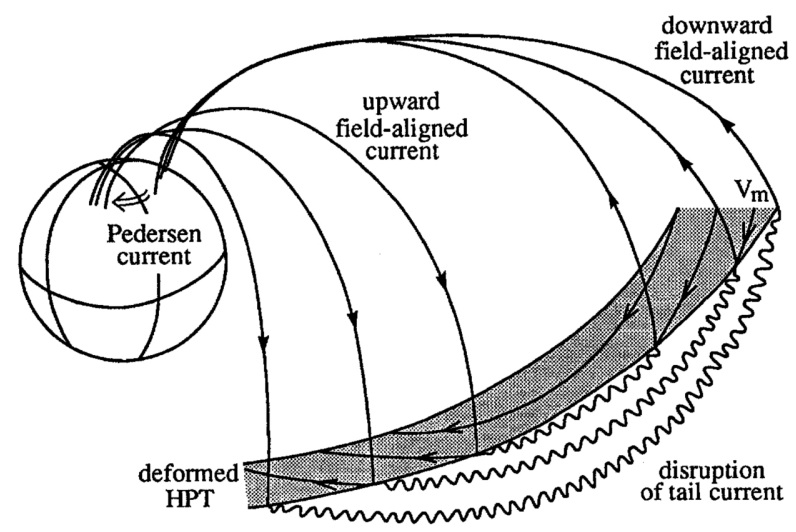

Fig. 8. Schematic representation of the tail current disruption and the consequent formation of a current wedge. The current disruption proposed in our model leads to enhancement of the region 1 FACs in azimuthally elongated regions. 
in Fig. 6(c) we find that a pair of the (postmidnight) downward and (premidnight) upward FACs, generated by the tail current disruption, are linked via the dawn to dusk Pedersen currents on the ionosphere. A so-called current wedge is then formed as is schematically shown in Fig. 8. From the simulation results, each region of downward and upward FACs enhanced by the current disruption is found to be azimuthally elongated, occupying an MLT range of at least a few hours, which are consistent with both the instantaneous (e.g., Richmond et al., 1988; Kamide et al., 1989) and statistical (e.g., Iijima and Potemra, 1976, 1978) plots of the FACs. It should be emphasized that such zonal distribution of the FACs is a natural result of the current disruption proposed in our paper. The present model for the current disruption is electrostatic, namely, a possible change in the magnetic field configuration is neglected. In Appendix $\mathrm{C}$, although the magnetostatic approximation is adopted, we show that the above-described current disruption can also occur when the associated change of the magnetic field is taken into account. (Furthermore, in Appendix D we estimate quantitatively, but within the order of magnitude, the effect of the field line deflection caused by FACs, which may affect, in some degree, the FAC generation studied in our paper.)

In passing, note that within our knowledge this type of the current disruption has never been recognized by previous authors. So far, the tail current disruption has theoretically been discussed only from the viewpoint of the magnetic reconnection. Within a frame of the reconnectionassociated current disruption which is likely to occur at the open-close boundary, it is difficult to understand the enhancement of region 1 FACs well inside the open-close boundary, as has been observed from the geosynchronous satellites (e.g., Nagai, 1982; Ohtani et al., 1990). Basically, as has been discussed in the introduction of Yamamoto et al. (1996), the reconnection-produced FACs are confined to a narrow $\left(\sim 0.3^{\circ}\right)$ latitudinal extent on the ionospheric plane so that they do not correspond to the large-scale FACs.

\section{$3.3 \quad$ Run 3}

The results of run 3 are presented in Figs. 9 and 10. Figure $9(\mathrm{a})$ shows the density $\left(n_{\mathrm{i}}\right)$ profile of the HPT at the time of about $10 \mathrm{~min}$. Figure 9 (b) shows the field-aligned current density resulting from the HPT deformation in Fig. 9(a). Figure 9(c) shows the internal-potential at $t \sim 10$ min. Figures 10(a) and 10(b) show the temporal variations of the maximum (region 1 ) FAC density and the internal-potential drop across the polar cap, respectively.

Since the external-(solar wind) convection is not included in this run, one can see how the internal-field develops by itself when the deformed HPT, finally obtained in run 1, is "initially" given. As is already discussed above, the internal-field can further deform the HPT such that intensification of the FACs (particularly the region 1 FACs) results. Such self-development of the internal-field and the FACs is confirmed by the results of Fig. 10. We can then suggest a possibility that the paired region 1 /region $2 \mathrm{FACs}$, or equivalently, the internal-convection with twin vortex cells is maintained by itself, for some time, even after the IMF $B_{Z}$ vanishes or turns northward. (Under these IMF conditions, the solar wind is thought to have little influence on the HPT dynamics.)

Even after the external-convection is switched off, the generator for the region $1 /$ region 2 FACs can continue working for some time. Basically, electrical energy is generated at the expense of kinetic energy of the HPT particles (i.e., $\boldsymbol{J}_{\perp} \cdot \boldsymbol{E}<0$ ), and it is transmitted, as a Poynting flux, to the ionosphere, where it is finally dissipated as Joule heating. The total power supplied from the generator must be balanced with that dissipated in the ionosphere. In our simulations the average value, $P_{\mathrm{i}}$, of the dissipated power per unit area on the ionospheric plane is estimated as $P_{\mathrm{i}}=\Sigma_{\mathrm{P}} E_{\mathrm{i}}^{2} \sim 8 \mathrm{mho} \cdot(15 \mathrm{mV} / \mathrm{m})^{2} \sim 1.8 \times 10^{-3} \mathrm{~W} / \mathrm{m}^{2}$. On the other hand, the total particle energy in the flux tube is given by $\left(W^{p}+W^{e}\right) N=\left(W^{p}+W^{e}\right) n R_{B} B_{\mathrm{i}}$, which is, on average, $\sim 50 \mathrm{~J} / \mathrm{m}^{2}$. The particle kinetic energy is also lost by electron precipitation; the average electron energy flux at the ionospheric height is about $8 \times 10^{-4} \mathrm{~W} / \mathrm{m}^{2}$. The total loss rate of the particle 


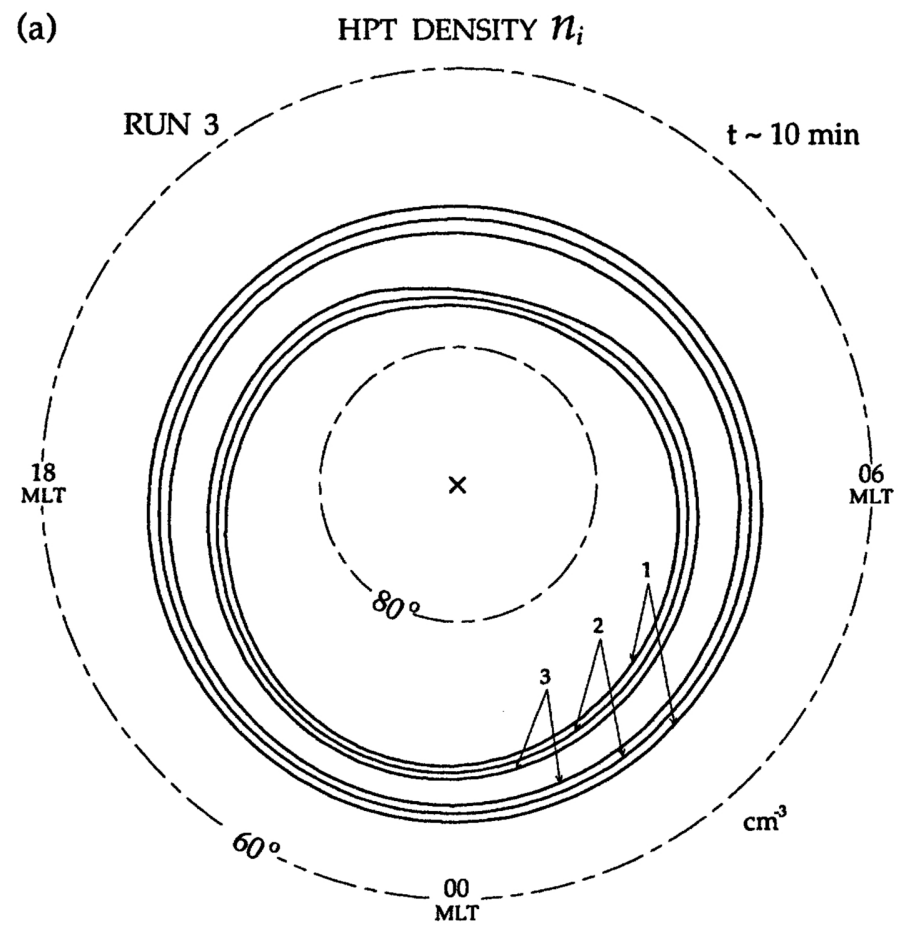

(b) FIELD-ALIGNED CURRENT DENSITY (magnetic drift)

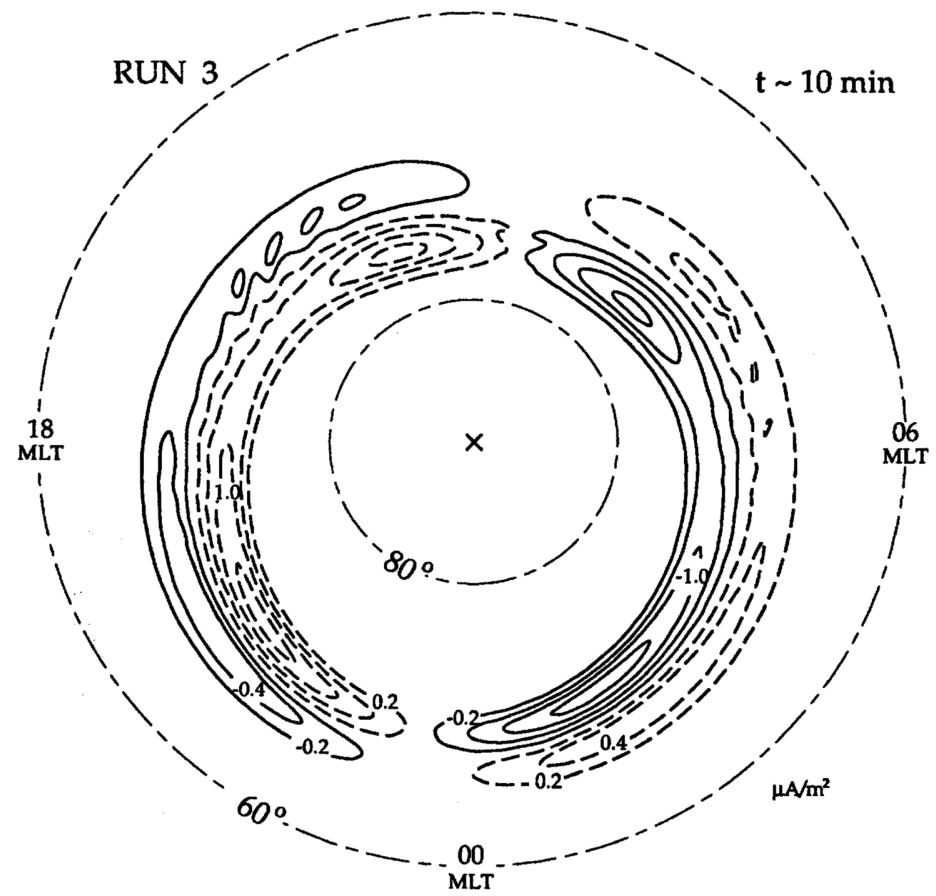

Fig. 9. Results of run 3 at $t \sim 10 \mathrm{~min}$, in the same format as Figs. 4(a), 4(c) and 4(d), but the contour interval in the potential profile (c) is $2.5 \mathrm{kV}$. 
(c) INTERNAL-POTENTIAL

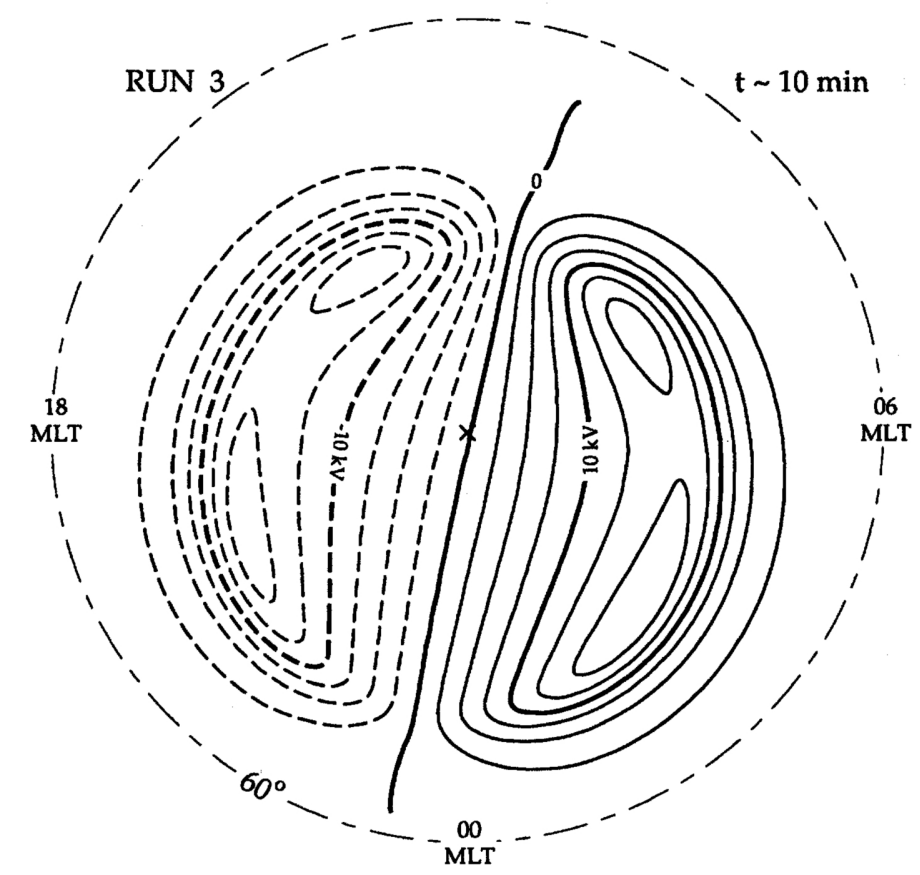

Fig. 9. (continued).

energy is then about $2.6 \times 10^{-3} \mathrm{~W} / \mathrm{m}^{2}$. Consequently, under the relatively quiet condition such as the electric field $E_{\mathrm{i}}$ on the ionosphere is of the order of $10 \mathrm{mV} / \mathrm{m}$, the generator of the HPT particles could continue working for a few hours, even if energy is not supplied externally. In the disturbed period such that $E_{\mathrm{i}}$ is increased to more than several tens of millivolts per meter, the ionospheric Joule heating would significantly dissipate the HPT kinetic energy (which is responsible for the FAC generation) on the time scale of a few tens of minutes. In this case the kinetic energy supply from, for example, the magnetic reconnection (see Appendix A) is vital for maintenance of intense FACs. (The energy equation for the HPT particles is neglected in the present simulations. This neglect (of $\boldsymbol{J} \cdot \boldsymbol{E}<0$ in the HPT) is partly responsible for the fast growth of the FAC intensities and the polar cap potential drop as shown in Figs. 5 and 10. Another important process controlling the growth of the FAC/potential is the particle injection into the nightside closed region, which will be discussed later.)

In considering the long-term ( $>20 \mathrm{~min}$ ) behavior of the paired region $1 /$ region $2 \mathrm{FAC}$ system after a geomagnetic storm, we need take into account the "flywheel" effect of the neutral wind as a result of the thermosphere-magnetosphere coupling (Lyons et al., 1985). Notably, Wygant et al. (1983) have shown, on the basis of the S3-3 satellite observations, that the polar cap potential drop during the periods of northward IMF depends on how long the IMF $B_{Z}$ had been northward previous to the measurement. The scatter plot of the measured polar cap potential versus the duration time of the northward IMF (Fig. 6 of Wygant et al.) is shown as Fig. 11 in our text. Interestingly, as has been suggested by Wygant et al., the polar cap potential appears to exponentially decay with the characteristic time of several hours after the IMF turns northward. Combining two important concepts of the HPT distortion leading to the region 1/region 2 FAC generation and the neutral wind flywheel effect, Yamamoto and Ozaki (1994) have proposed a 
(a) MAXIMUM FAC DENSITY

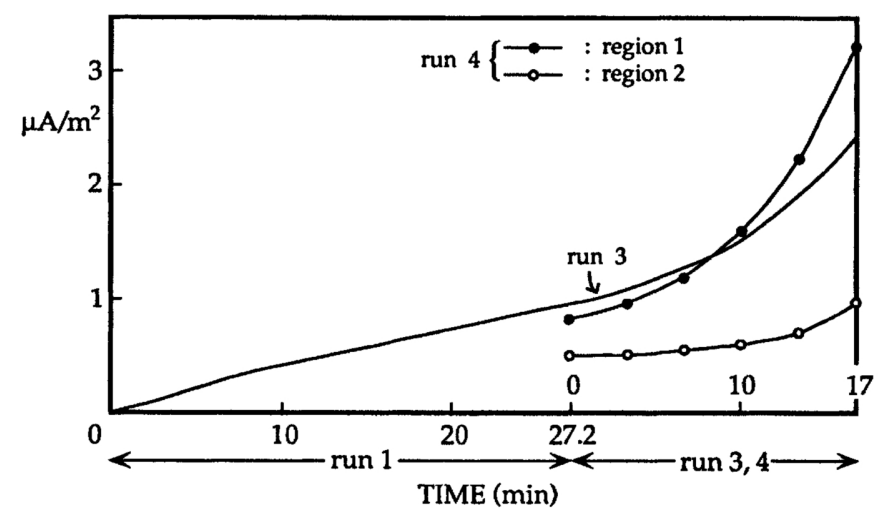

(b) POLAR CAP POTENTIAL DROP OF INTERNAL-FIELD

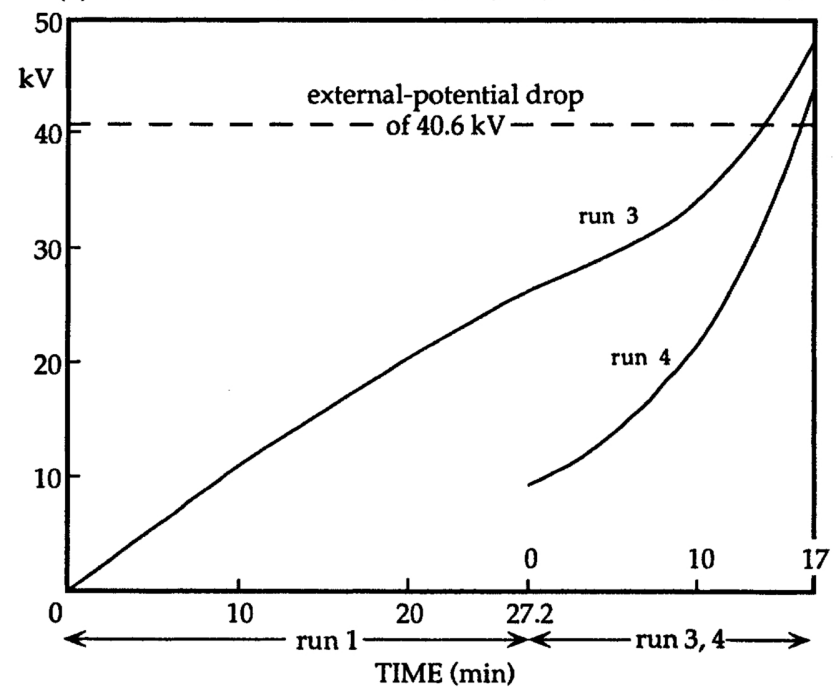

Fig. 10. (a) Temporal variations of the maximum (region 1) FAC density in the consecutive runs 1 and 3 , and the maximum region 1 and region 2 FAC densities in run 4. (b) Temporal variations of the (internal-) potential drops across the polar cap in the consecutive runs 1 and 3 , and in run 4 .

possible scenario to explain the many hours endurance of the polar cap potential as well as the region 1/region 2 FACs after a geomagnetic storm. This model is briefly described in Appendix E.

\subsection{Run 4}

The simulation results of run 4 are presented in Figs. 10 and 12. Figures 10(a) and 10(b) show the temporal variations of the maximum region 1 and region 2 FAC densities and the internalpotential drop across the polar cap, respectively. Figure 12(a) shows the density $\left(n_{\mathrm{i}}\right)$ profiles of the HPT "initially" given and that at $t \sim 10 \mathrm{~min}$. Recall that the "initial" HPT configuration in this run is obtained by impressing the solar wind convection upon the HPT with the midnightdirected density gradient (Fig. 1(c)) for the time period of about $27 \mathrm{~min}$. Figure 12(b) shows the field-aligned current densities resulting from the HPT deformation in Fig. 12(a). Figure 12(c) shows the corresponding internal-potential. 


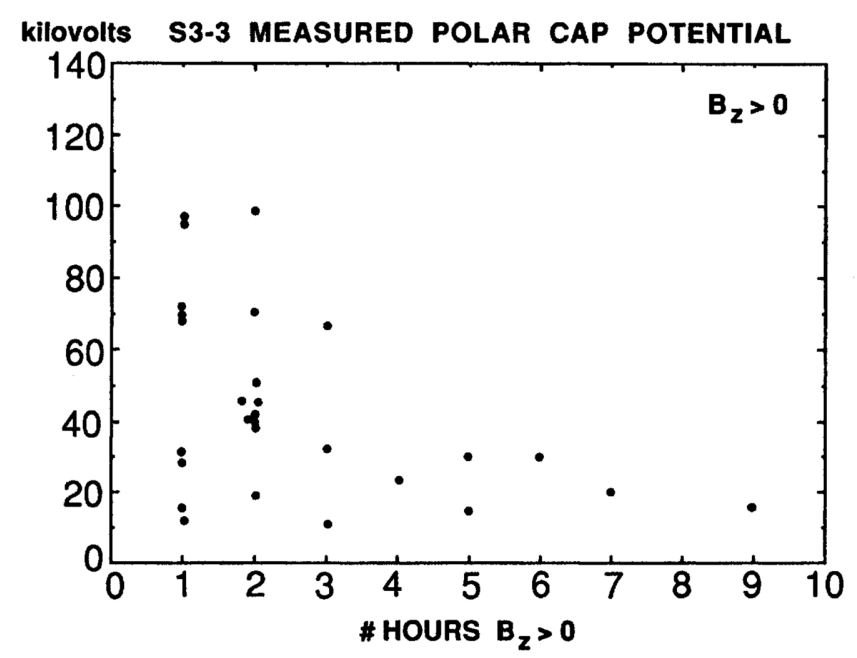

Fig. 11. Scatter plot of the measured polar cap potential drop versus the duration time that the IMF $B_{Z}$ had been northward previous to the measurement (from Fig. 6 of Wygant et al. (1983)).

As has commonly been accepted, the azimuthal pressure gradient toward local midnight can enhance the region 2 FACs due to the opposite directions of the magnetic drifts of ions and electrons. (Such pressure gradient might be due to the effect of the particle injection and/or energization occurring in the midnight sector.) The enhancement of the region 2 FACs is confirmed by comparing the FAC distribution (at $t=0$ ) in Fig. 12(b) and that in Fig. 4(c) (run 1). (Note that the difference between these two FAC distributions comes from only the midnight-directed pressure gradient initially given.) At the same time, the region 1 FAC (at $t=0$ ) in Fig. 12(b) is appreciably decreased by the effect of the midnight-directed pressure gradient, compared with that in Fig. 4(c).

From Fig. 12(c) we find that the regions of positive and negative potentials emerge in the dusk and dawn sectors, respectively, corresponding to the enhancement of the region 2 FACs. The development of the region 2-associated potential reduces the polar cap potential drop. On the other hand, the intensification of a pair of the region 1 FACs in the premidnight and postmidnight sectors (as is already discussed for runs 2 and 3) occurs in the course of run 4. Simultaneously with this process, the (region 1-associated) two-cell potential is splitted into the nightside and dayside parts (see Fig. 12(c)). The region 1 FAC intensification (on the nightside) may be promoted by the isolation of the associated two-cell convection. The splitting of the region 1-associated potential may in turn be facilitated by the appearance of the region 2-associated potential with opposite polarity (compare Figs. 9(c) and 12(c)). Thus we can understand that the region 1 FACs in run 4 , in the later period, increase faster than those in run 3 (see Fig. 10(a)).

In summary, the paired region 1/region 2 FAC system has the tendency toward splitting, partializing a quadruplet of FACs (region 1 pair plus region 2 pair) as seen in Fig. 12(b) (at $t \sim 10$ min). The local development of quadruple FACs, particularly in the premidnight/postmidnight sectors, can sometimes be seen in the instantaneous plots of the observed FACs (e.g., Richmond et al., 1988; Kamide et al., 1989). As an example of the quadruple FAC system, Fig. 4 of Richmond et al. (1988) is reproduced as Fig. 13 in our text. The FAC pattern in this figure is similar to that from our simulations (e.g., at $t \sim 10 \mathrm{~min}$ in run 4). Only the significant difference is the triple structure of the observed FACs (upward FAC zone sandwiched between two downward FAC zones) in the premidnight sector. A possible formation mechanism for the triple FAC structure 
(a) HPT DENSITY $n_{i}$

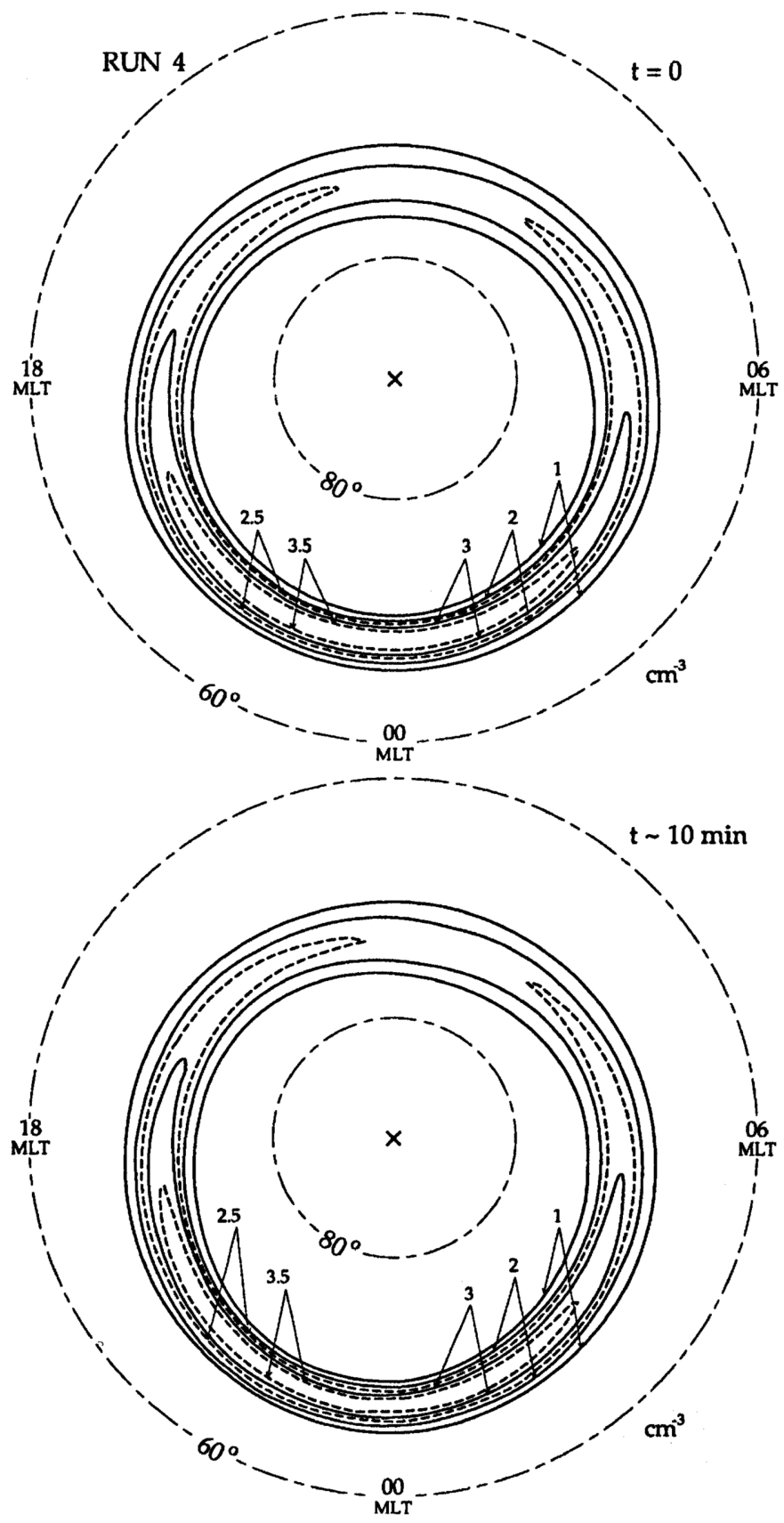

Fig. 12. Results of run 4 at $t=0$ and $t \sim 10 \mathrm{~min}$, in the same format as Figs. $4(\mathrm{a}), 4(\mathrm{c})$ and $4(\mathrm{~d})$, but the contour interval in the potential profile (c) is $1 \mathrm{kV}$. 
(b) FIELD-ALIGNED CURRENT DENSITY (magnetic drift)

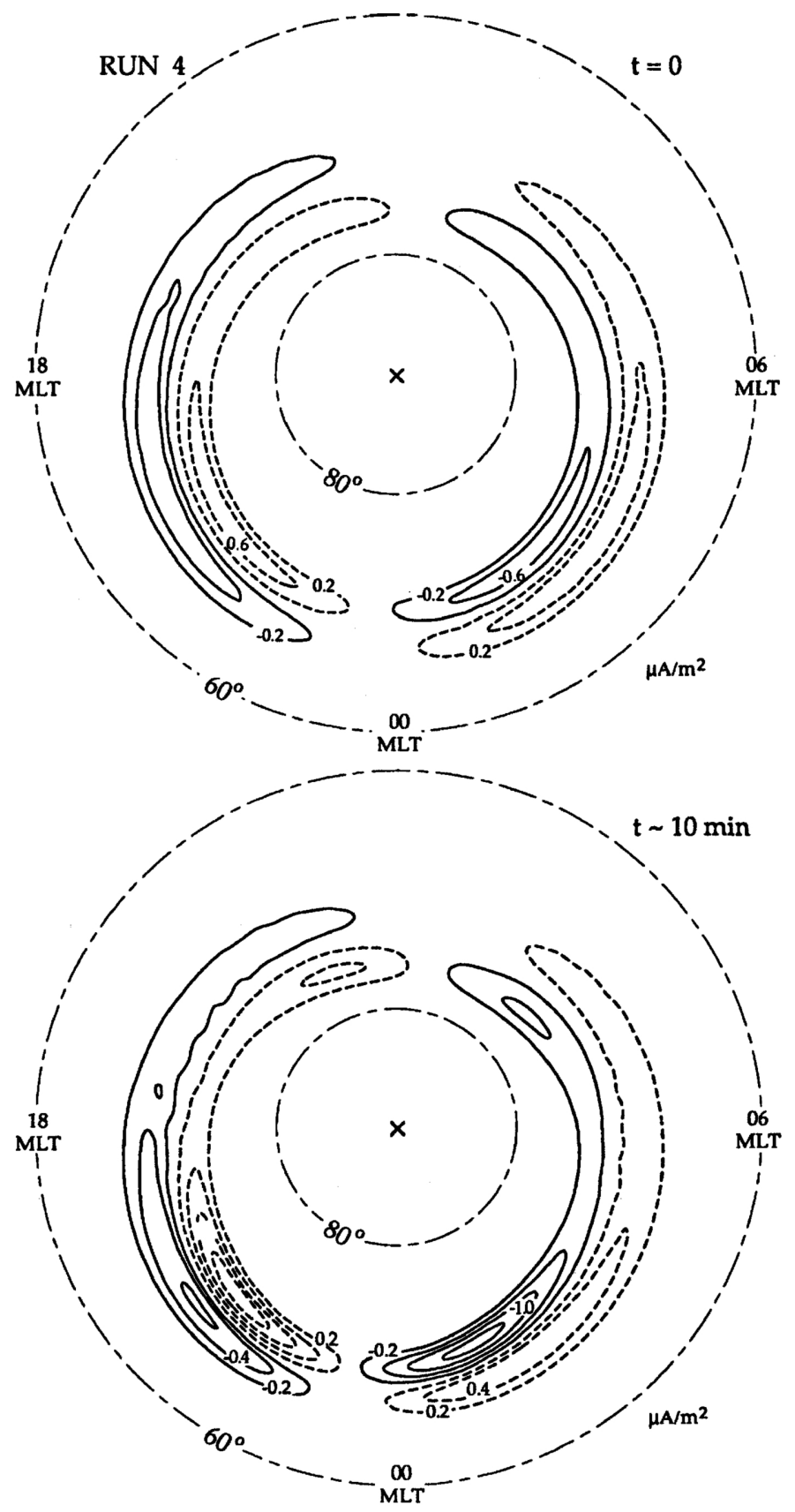

Fig. 12. (continued). 


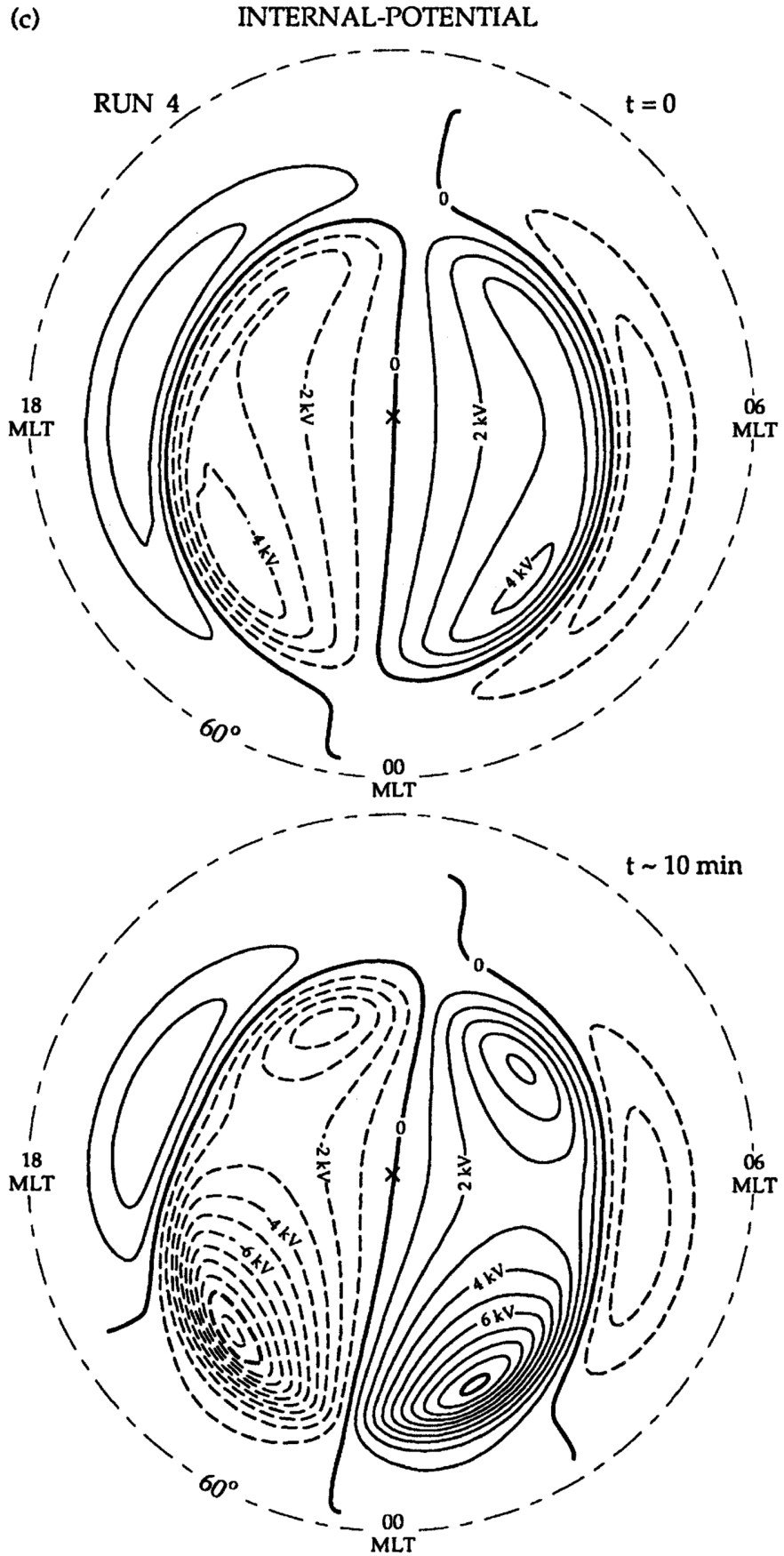

Fig. 12. (continued). 


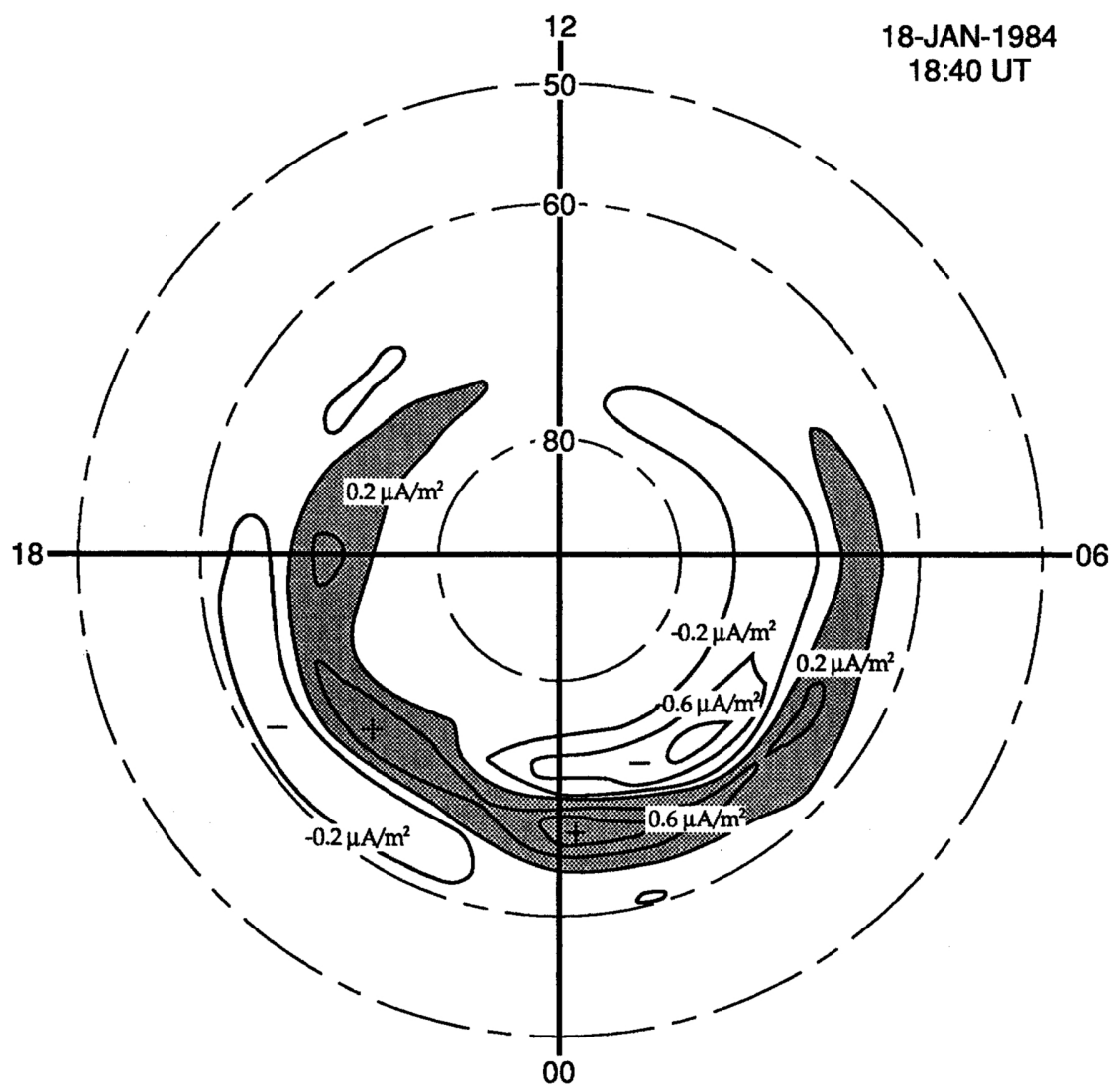

Fig. 13. Instantaneous plot of FAC densities on the ionosphere nearly at the maximum epoch of a substorm, which was obtained by Richmond et al. (1988) on the basis of the combined incoherent-scatter radar and ground magnetometer observations. The field-aligned currents are upward in the shaded region.

is studied by Yamamoto et al. (1997b).

In passing, note that the development of a quadruplet of FACs is accompanied by the generation of westward and eastward plasma jets around the interface between the regions of upward and downward FACs. Actually, in the simulations, the speed of the azimuthal jets on the ionosphere reaches as large as about $0.5 \mathrm{~km} / \mathrm{s}$, e.g., at $t \sim 10 \mathrm{~min}$ in run 4 (Fig. 12(c)). Such generation of plasma flows, for example, in the premidnight/postmidnight sectors, gives us the impression as if plasmas were ejected in the azimuthal direction by some special mechanism acting around local midnight. In the model by Rostoker and Boström (1976), the azimuthal jets were thought to be the cause of the paired region 1 /region 2 FACs. On the contrary, our conclusion in the present study is that the production of the azimuthal jets is a result of the generation of the paired region 1 /region 2 FACs arising from the distortion of the HPT in the magnetosphere.

In concluding this section we consider possible effects of particle injection into the nightside closed region, which is neglected in our simulations. As is discussed in Appendix A, the particle injection is assumed to occur in the premidnight to postmidnight sector. Newly injected particles are then thought to occupy some region which is just poleward of the preexisting HPT. In the 
presence of a two-cell convection, the injected particles will follow up the HPT transported to lower latitudes. Namely, the newly injected particles are supplied primarily to an MLT sector where the number of the preexisting HPT particles is decreased owing to the convection flow bifurcation into the westward and eastward directions (see, e.g., Fig. 7). As a result, the development of the region $1 \mathrm{FACs}$ in the premidnight to postmidnight sector tends to be suppressed. If particles injected from outside of the closed region are included into the simulations, the region 1 FACs as well as the polar cap potential drop of the internal-field will not be developed so fast as shown in Figs. 5 and 10. The observation-based study by Kamide and Baumjohann (1985) (see Fig. 5 of their paper) has shown that the polar cap potential drop varies severely during the substorm periods: even a potential change of $\sim 100 \mathrm{kV}$ within a few tens of minutes is not uncommon, which suggests that the FAC system could be quite unstable. For a quantitative description of such behavior of the magnetosphere/ionosphere system during disturbed periods, we should self-consistently treat the particle injection, developing a more global simulation model to cover both of the closed and open regions. In other words, our future task is to systematically study the formation and stability of the HPT, from a global viewpoint of the interaction between the solar wind and the total system of the magnetosphere/ionosphere.

\section{Conclusions}

By the two-dimensional numerical simulations with ionospheric coupling, we have demonstrated that the paired region 1 and region 2 field-aligned currents can be generated as a result of "natural distortion" of the hot plasma torus (HPT) in the magnetosphere under the influence of the usually prevailing convection with twin vortex cells (when IMF $B_{Z}<0$ ). (If the effect of the anomalous cross-field diffusion on the HPT polarization is included, the resulting FAC pattern with the triple structure in the midnight sector remarkably agrees with the observations (Yamamoto et al., 1997b).) New important findings to finally emphasize are in the following.

1. Persistent coupling between the region 1 and region 2 FACs, which has been evidenced by the observations, can essentially be attributed to the polarization of the single topological structure, that is, the hot plasma torus in the magnetosphere.

2. The tail current disruption, which has been observed to occur over a wide MLT range and well inside the closed region, can be understood as a natural result of the HPT distortion on the nightside, which further progresses in the region 1 FAC zone than in the region 2 zone.

The work of T. Yamamoto was supported in part by the Ministry of Education, Japan, grant 07804029 as well as the joint research programs of Radio Atmospheric Science Center, Kyoto University, Uji, Kyoto and the Institute of Space and Astronautical Science, Sagamihara, Kanagawa. The editor thanks T. Tanaka and another referee for their assistance in evaluating this paper.

\section{Appendix A}

In this appendix we study a possible scenario for the formation of the hot plasma torus (HPT) in the magnetosphere, when the IMF $B_{Z}$ is negative so that the open/close boundary is clearly defined. There are three basic processes which might be important for the HPT formation: 1 . nonadiabatic acceleration of ions incident on the tail current sheet (including particle acceleration in the magnetic reconnection), 2. plasma escape, along the open field lines, to the interplanetary space, and 3. anomalous cross-field diffusion of plasma particles.

1. Lyons and Speiser (1982) have shown that ions incident on the tail current sheet can be nonadiabatically accelerated due to the dawn to dusk electric field; the characteristic particle energy is increased from several hundred electron volts to a few kilo electron volts (see Figs. 2, 3, 9 
and 10 of their paper). The velocity distribution of accelerated ions which results from the current sheet acceleration was also shown to be consistent with the observations at the outer edge of the plasma sheet. Since the ion beams are frequently observed, by the midaltitude Akebono satellite, in a relatively wide range of 20-04 MLT (Saito et al., 1992), the current sheet acceleration in the tail may be regarded as an important energy source of plasma sheet ions.

Similar ion acceleration can occur in the reconnection process. As reconnection proceeds, the tail current around the neutral point is significantly decreased within several minutes (tail current disruption) (e.g., Sato, 1982). In the course of current disruption, westward inductive electric field is enhanced in the reconnection region. Signatures of such electric field enhancement $0 \lesssim 10 \mathrm{mV} / \mathrm{m}$ have been detected from, for example, the ISEE-1 satellite in the near Earth $\left(X \sim-20 R_{\mathrm{E}}\right.$ ) tail (Cattell and Mozer, 1984). Hence ions can substantially be accelerated along the neutral line by the westward inductive electric field. According to the numerical calculation of test particle trajectories in developing reconnection fields (Sato et al., 1982), ions can be accelerated near the neutral line to $1-200 \mathrm{keV}$, depending on how close they reach the neutral line after injection. The thus accelerated particles eventually turn to the field-aligned plasma flow. Note that the magnetic reconnection is thought to be a powerful energy converter which transforms magnetic energy into plasma flow and thermal energy, particularly in the substorm periods.

2. In the presence of a two-cell convection, the thus energized ions are transported to the dayside, eventually getting out of the closed region. Ions on the open field lines will escape into the interplanetary space. Consequently, just outside of the closed region the flux tube content,

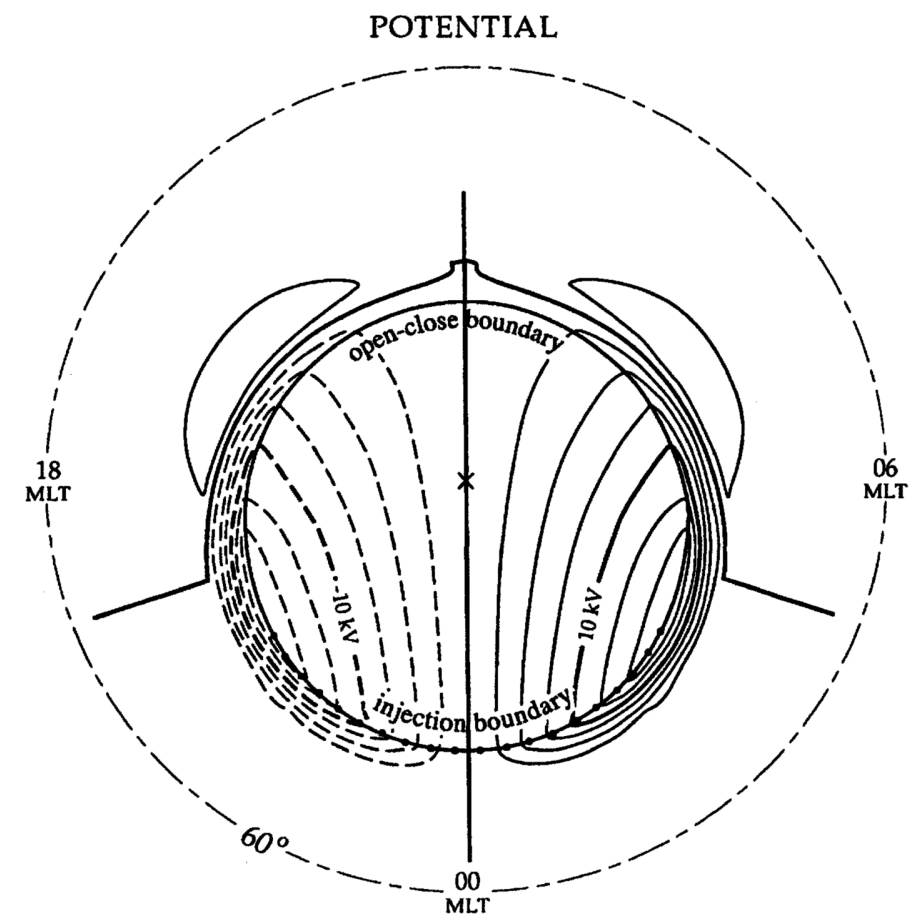

Fig. 14. A typical pattern of the $E \times B$ convection projected onto the polar ionosphere. The assumed open-close boundary is indicated. Some part of the open-close boundary where the inward (toward lower latitudes) flow occurs is referred to as the injection boundary. 


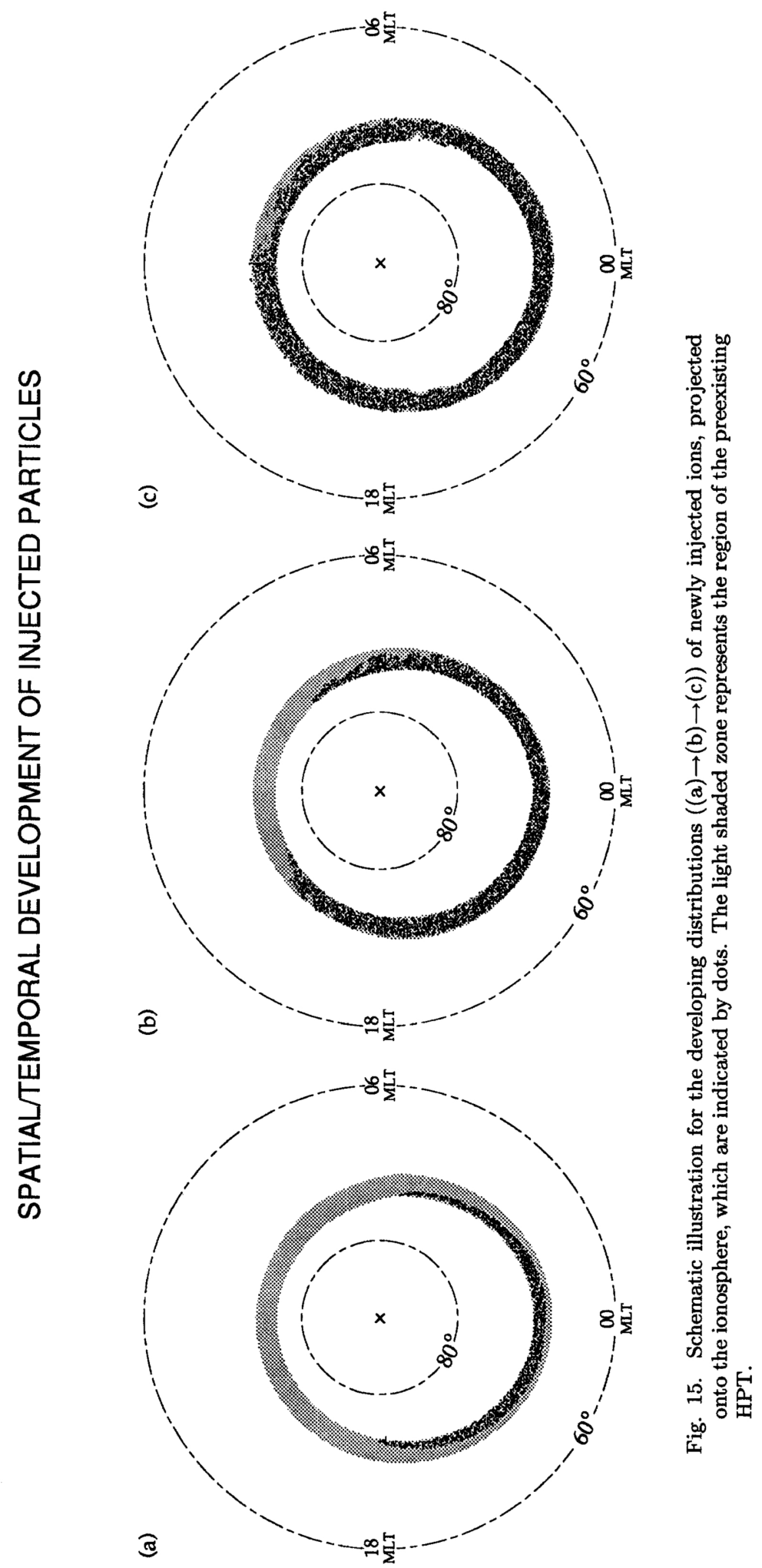


$N$, of a plasma in the near Earth region is decreased with the distance from the open/close boundary. The characteristic distance, $d$, for the decrease in $N$ is estimated as follows. The time required for escaping is roughly calculated as $\ell_{\|} / v_{\|} \sim 30 R_{\mathrm{E}} / 500 \mathrm{~km} \mathrm{~s}^{-1} \sim 380 \mathrm{~s}$, where $\ell_{\|}$is the characteristic length of the last closed field line, and $v_{\|}$is the typical field-aligned speed of the ions. As the radial speed of the large-scale convection is usually a few hundred meters per second, the decay length $d$ is found to be about $100 \mathrm{~km}$.

3. On the basis of the satellite observations of low-frequency waves in the auroral magnetosphere, Yamamoto et al. (1997b) have estimated the anomalous cross-field diffusion rate in a frame of quasi-linear theory. Likely candidates for wave turbulence causing the anomalous diffusion are the Alfvén wave noise (e.g., Gurnett et al., 1984; Block and Fälthammar, 1990; Lundin et al., 1990; Chmyrev et al., 1985; Dubinin et al., 1985, 1988) and the broadband electrostatic noise (e.g., Scarf et al., 1974; Gurnett et al., 1976; Gurnett and Frank, 1977). (For a brief review, see Yamamoto et al. (1997b).) They have also shown that the ion diffusion coefficient averaged over the flux tube can reach a significant fraction of the Bohm rate at least in disturbed periods.

In the presence of a two-cell convection as shown in Fig. 14, the plasma particles are injected from some part of the nightside open/close boundary. The injection occurs where the convection flow has an inward radial component. Such a part of the open/close boundary may be called the injection boundary (see Fig. 14). Due to the current sheet acceleration, i.e., process 1, the ions are assumed to be energized, typically, to a few kilo electron volts immediately after injection. Figure 15 schematically shows how the distribution of injected and energized ions evolves with time, where the density of dots is proportional to the flux tube content of the newly injected ions, and the shaded zone represents the preexisting HPT. Note that, due to process 2 , i.e., escape to interplanetary space, the density distribution has a (relatively) sharp cutoff at the open/close boundary where the particles are exiting out of the closed region. Circling of the distribution of injected ions (see Fig. 15(c)) will asymptotically be attained by the aid of the $E \times B$ drift flow which arises, in the noon (or prenoon) sector, due to charge separation in the Alfvén layer. Figure 16(a) shows the distribution of the flux tube content $N$ for all the particle population (i.e., both of the preexisting and newly injected ions): the left panel shows the two-dimensional profile of $N$ corresponding to Fig. 15(b), and its latitudinal profile is on the right. If the effect of the anomalous cross-field diffusion (process 3 ) is included, primarily the radial (latitudinal) profile of $N$ is significantly modified so that the plateau shape is changed to a simply peaked one (although a sharp cutoff still remains near the open/close boundary). Figure 16(b) schematically shows the distribution (in $N$ ) of the HPT ions when the radial diffusion significantly proceeds in the distribution of Fig. 16(a).

We assume that precipitation of ions is negligible compared with that of electrons, in other words, field-aligned currents are predominantly carried by electrons. From the condition of charge neutrality, the ion distribution in $N$ as shown in Fig. 16 can then be identified with the electron distribution. In conclusion the HPT as in Fig. 16 is likely to be formed, through processes 1-3, in the presence of a two-cell convection when the IMF has a southward component.

\section{Appendix B}

In this appendix we discuss the density $(n)$ distribution of the HPT in the magnetosphere, when the HPT distortion occurs, being responsible for the generation of the paired region $1 /$ region 2 FACs. Recall that the density $n\left(=N / R_{B} B_{\mathrm{i}}\right)$ is different from the density $n_{\mathrm{i}}\left(=N /\left(R_{B} B_{\mathrm{i}}\right)_{0}\right)$ of the virtual particles used in the simulations, where $N$ is the flux tube content ("surface" density) of the HPT particles and $\left(R_{B} B_{\mathrm{i}}\right)_{0}$ is evaluated for the dipole field line at $L=7 R_{\mathrm{E}}$ (see Section 2). As an example, in Fig. 4(b) we show the two-dimensional pattern (upper panel) and the latitudinal (in $x$ ) profile along the midnight meridian (lower panel) of the HPT density 


\section{(a) FLUX TUBE CONTENT, $N$ (without diffusion )}
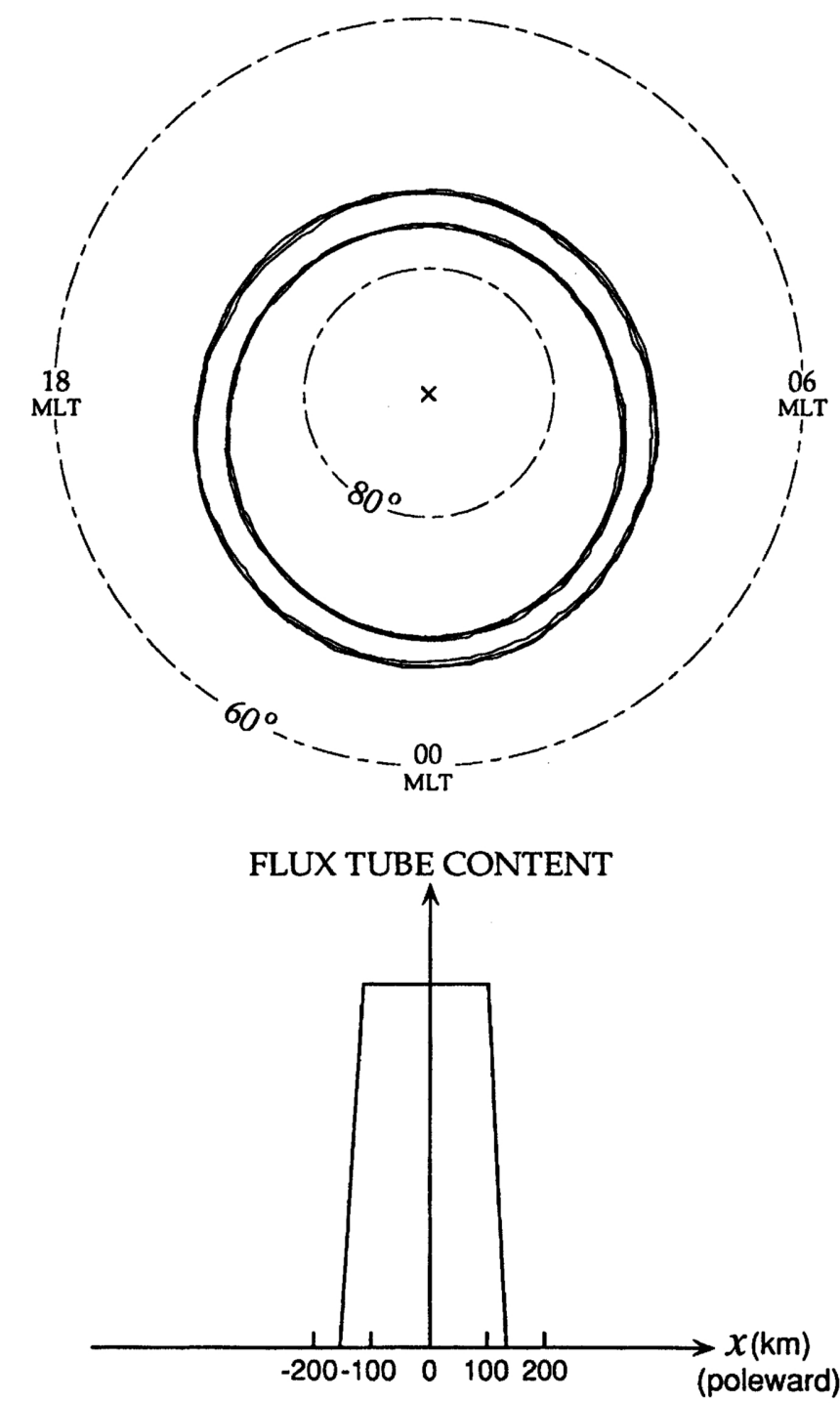

Fig. 16. (a) Equicontours (in left panel) of the flux tube content $N$ for all the particle population (i.e., both of the preexisting and newly injected ions) which is indicated in Fig. 15(b). The latitudinal profile of $N$ is on the right. (b) Distribution (in $N$ ) of the HPT ions when the radial diffusion significantly proceeds in the distribution in (a).

$n$, which corresponds to the density $n_{\mathrm{i}}$ of the distorted HPT (Fig. 4(a)) obtained in run 1. For comparison, in Fig. 4(b) the profile $n_{\mathrm{i}}(x)$ is also indicated by the dashed line.

Since $R_{B}$ is constant along the magnetic drift path (see Section 2 of Yamamoto et al. (1996)), the azimuthal (in the magnetic drift direction) gradient of the density $n$ is found to be different, by the factor of $\left(R_{B} B_{\mathrm{i}}\right)_{0} / R_{B} B_{\mathrm{i}}$, from that of the density $n_{\mathrm{i}}$. As is seen from Fig. 7 , on the nightside, the HPT is convected to lower latitudes while it is being deformed. Since the flux tube volume $R_{B}$ is smaller at lower latitudes (see Fig. 1(b)), in terms of the density $n$, the midnight- 
(b) FLUX TUBE CONTENT, $N$ (after diffusion)
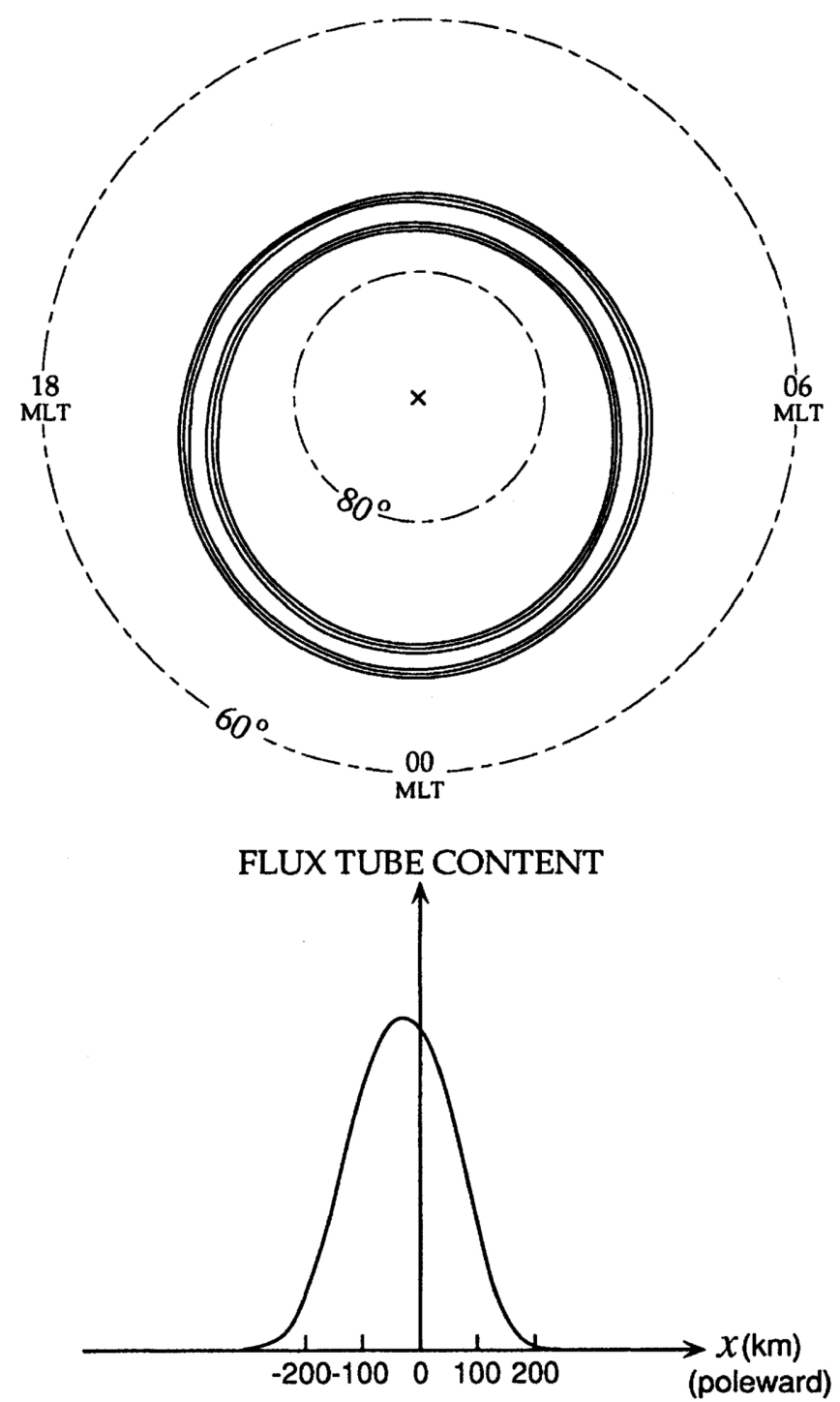

Fig. 16. (continued).

directed azimuthal density gradient of the deformed HPT in the region 2 zone on the nightside will be more striking, compared with the reverse azimuthal density gradient in the counterpart of the region 1 FAC zone (see Fig. 4(b)). Here note that the dominance of the region 2 in the magnitude of density gradient does not mean the region 2 FAC density greater (in magnitude) than the region $1 \mathrm{FAC}$ density because the dominance in the density gradient is cancelled by the smaller value of $R_{B}$ in the region 2 FAC zone (see Section 4 of Yamamoto et al.). Finally it should be noted that in the latitudinal density profile of Fig. 4(b), the density $n$ is peaked inside the region 2 FAC zone while the density $n_{\mathrm{i}}$ is peaked around the interface between the region 1 and region 2 FACs (see Section 2 of Yamamoto et al.). 


\section{Appendix C}

In this appendix, under the magnetostatic approximation, we discuss how the process of tail current disruption proposed in Section 3 is modified by the associated change of the magnetic field configuration, which is not included in the electrostatic model of our simulation. Specifically we argue that on the ionospheric plane the flux tube volume $R_{B}$ (defined in Section 2) on an equicontour of the flux tube content $N$ (see Eq. (3)) will attain azimuthal (away from midnight) gradients while the plasma is inwardly (toward the Earth) transported under the influence of a two-cell convection. Suppose that initially the equicontours of $N$ are aligned with those of $R_{B}$ so that no FAC is generated (see Eq. (7)). (Recall that the average magnetic drift velocity $\bar{V}_{\mathrm{m}, \mathrm{i}}$ as projected onto the ionospheric plane (see Section 2) is tangent to the $R_{B}$-contour (see, e.g., Appendix B of Yamamoto et al. (1996)):

$$
\overline{\boldsymbol{V}}_{\mathbf{m}, \mathrm{i}}=\frac{2 W}{3 q R_{B} B_{\mathrm{i}}^{2}} \nabla_{\mathrm{i}} R_{B} \times \boldsymbol{B}_{\mathrm{i}}
$$

where $q$ is the charge of a particle, $W$ is the average energy of the particles with isotropic pressure, and $\boldsymbol{B}_{\mathrm{i}}$ is the magnetic field on the ionosphere.) According to our simulation results, the $N$-contours on the nightside can be deformed as shown in Fig. 17, as a result of the inward convection. Here we consider the conservation of the ions in the flux tube, which is expressed as

$$
\frac{D}{D t} N \equiv\left\{\frac{\partial}{\partial t}+\left(\boldsymbol{V}_{\mathrm{E}, \mathrm{i}}+\overline{\boldsymbol{V}}_{\mathrm{m}, \mathrm{i}}\right) \cdot \nabla_{\mathrm{i}}\right\} N=0
$$

where $\boldsymbol{V}_{\mathrm{E}, \mathrm{i}}$ is the electric drift velocity on the ionospheric plane. (For the condition required for expression (C2), see Yamamoto et al. (1996).) Equation (C2) implies that an "ion fluid" carrying a flux tube content $N$ is convected with the velocity of $\boldsymbol{V}_{\mathrm{E}, \mathrm{i}}+\overline{\boldsymbol{V}}_{\mathrm{m}, \mathrm{i}}$ on the ionospheric plane. Let the position of an ion fluid be given by $r_{\mathrm{i}}(t)$. In what follows we evaluate the change $\left(\delta R_{B}\right)$ of the flux tube volume $R_{B}$ at the position of $\boldsymbol{r}_{\mathrm{i}}(t)$, when the ion fluid is displaced by $\delta r_{\mathrm{i}}$; namely $\delta R_{B}=R_{B}\left(\boldsymbol{r}_{\mathrm{i}}(t)+\delta \boldsymbol{r}_{\mathrm{i}}\right)-R_{B}\left(\boldsymbol{r}_{\mathrm{i}}(t)\right)$ for $\delta \boldsymbol{r}_{\mathrm{i}}=\boldsymbol{r}_{\mathrm{i}}(t+\delta t)-\boldsymbol{r}_{\mathrm{i}}(t)$.

For simple analysis, $R_{B}\left(r_{\mathrm{i}}\right)$ may formally be expressed as

$$
R_{B}\left(r_{\mathrm{i}}\right) \sim F\left(\xi\left(r_{\mathrm{i}}\right)\right) R_{B, \mathrm{~d}}\left(r_{\mathrm{i}}\right)
$$

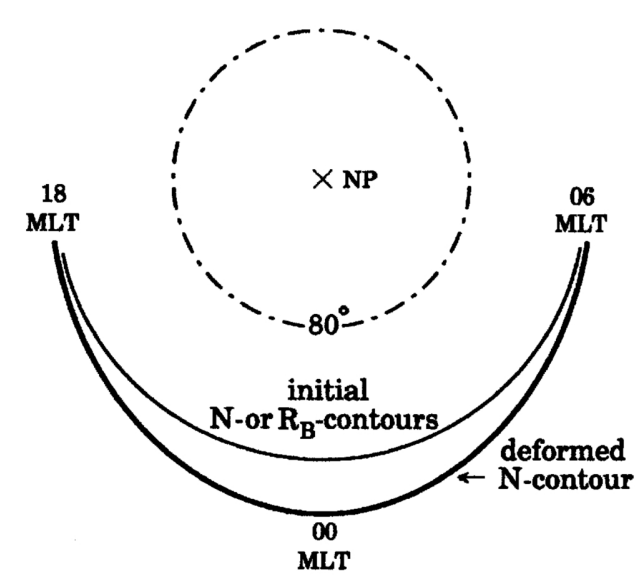

Fig. 17. Schematic illustration of a deformed equicontour (thick line) of the flux tube content $N$ on the nightside, relative to the initial contour (thin line) of $N$ or $R_{B}$ (flux tube volume). The deformation is assumed to result from the inward plasma convection. 
where $R_{B, \mathrm{~d}}$ is the volume of the dipole flux tube $\left(r_{\mathrm{i}}\right.$ specifies the ionospheric foot point of a dipole field line), and $F$ is the correction factor which depends on how the actual field line is stretched out away from the Earth or distended due to the presence of the tail current. Introducing a parameter, $\xi$, representing the degree of the field line distention, $F$ is regarded as an increasing function of $\xi$. The parameter $\xi$ could be related to the strength of the magnetic field produced from the tail current relative to that of the Earth dipole field. In practice, $\xi$ may simply be defined as $\xi=B_{0} / B_{\mathrm{d}}$, where $B_{0}$ is the maximum strength of the tail-current associated field along the field line with foot point at $\boldsymbol{r}_{\mathrm{i}}$, and $B_{\mathrm{d}}$ is the dipole field strength, at the equator, on the same field line. Thus the factor $F$, as a function of $\xi$, has the following characteristics:

$$
F(0)=1 \quad \text { and } \quad d F / d \xi>0 .
$$

The variation $\delta R_{B}$ defined above is now written as

$$
\delta R_{B} \sim F \delta r_{\mathrm{i}} \cdot \nabla_{\mathrm{i}} R_{B, \mathrm{~d}}+R_{B, \mathrm{~d}}(d F / d \xi) \delta \xi
$$

where $\delta \xi$ is the change of $\xi$ associated with the displacement $\delta \boldsymbol{r}_{\mathrm{i}}$. Using the local Cartesian coordinates $(x, y)$ on the ionosphere, where $x$ and $y$ correspond to the latitude and longitude, respectively, but they are scaled in distance, we rewrite the above equation as

$$
\delta R_{B} \sim F\left(d R_{B, \mathrm{~d}} / d x\right) \delta x_{\mathrm{i}}+R_{B, \mathrm{~d}}(d F / d \xi) \delta \xi .
$$

The variation $\delta \xi$ is formally expressed as

$$
\delta \xi=\xi\left(\delta B_{0} / B_{0}-\delta B_{\mathrm{d}} / B_{\mathrm{d}}\right) .
$$

Let the radial position of the intersection of a field line with the equatorial plane be given by the distance, $r_{\mathbf{e}}$, from the dipole axis. Then, as for the field line with foot point at $\boldsymbol{r}_{\mathrm{i}}$, the change of $r_{\mathrm{e}}$ corresponding to $\delta r_{\mathrm{i}}$ is designated by $\delta r_{\mathrm{e}}$. For the dipole field, $\delta B_{\mathrm{d}} / B_{\mathrm{d}}$ is expressed as

$$
\delta B_{\mathrm{d}} / B_{\mathrm{d}}=-3 \delta r_{\mathrm{e}} / r_{\mathrm{e}}
$$

The magnetic field $\boldsymbol{B}$ produced from the tail current with current density $\boldsymbol{J}$ can be related to the (isotropic) pressure, $p$, of a plasma carrying the tail current, using the equations of $\operatorname{rot} \boldsymbol{B}=\mu_{0} \boldsymbol{J}$ and $\boldsymbol{J}=\boldsymbol{B} \times \nabla p / B^{2}$. As an example, one can obtain a simple analytic solution for the twodimensional structure of the current sheet (e.g., Sato, 1979):

$$
\boldsymbol{B}=B_{0} \hat{X} \tanh \left(Z / L_{Z}\right), \quad \boldsymbol{J}=J_{0} \hat{Y} \operatorname{sech}^{2}\left(Z / L_{Z}\right), \quad p=p_{0} \operatorname{sech}^{2}\left(Z / L_{Z}\right), \quad p_{0}=B_{0}^{2} / 2 \mu_{0}
$$

where Cartesian coordinates $(X, Y, Z)$ are used, and $\hat{X}, \hat{Y}$ and $\hat{Z}$ are the unit vectors along the $X, Y$ and $Z$ axes, respectively; $L_{Z}$ represents the thickness of the current sheet. Similarly to this example, we assume the relation of $p_{0} \sim B_{0}^{2} / 2 \mu_{0}: p_{0}$ is the plasma pressure at the equatorial position nearest to the point where $B_{0}$ (which is again, as originally defined, the maximum strength of the tail-current produced field on a fixed field line) is found. Consequently we obtain

$$
\delta B_{0} / B_{0} \sim \delta p_{0} / 2 p_{0} .
$$

Consider the adiabatic plasma compression under the conservation of the flux tube content $N$ (as assumed above): if $N$ is expressed as $N=n R_{B} B_{\mathrm{i}}$ where $n$ is the plasma density uniform along the field lines, we have

$$
\delta n / n=-\delta R_{B} / R_{B}
$$

Assuming $p n^{-\gamma}=$ const, where $\gamma$ is the ratio of specific heats, combination of Eqs. (C8) and (C9) yields

$$
\delta B_{0} / B_{0} \sim-(\gamma / 2) \delta R_{B} / R_{B}
$$


From Eqs. (C6), (C7) and (C10), $\delta R_{B}$ in Eq. (C5) can be written as

$$
\delta R_{B} \sim F\left(d R_{B, \mathrm{~d}} / d x\right) \delta x_{\mathrm{i}}+R_{B, \mathrm{~d}}(d F / d \xi) \xi\left\{3 \delta r_{\mathrm{e}} / r_{\mathrm{e}}-(\gamma / 2) \delta R_{B} / R_{B}\right\} .
$$

Solving the above equation for $\delta R_{B}$, we obtain the expression for $\delta R_{B}$ :

$$
\delta R_{B} \sim\left\{\frac{F d R_{B, \mathrm{~d}} / d x+\left(3 / r_{\mathrm{e}}\right) \xi(d F / d \xi) R_{B, \mathrm{~d}}\left(\delta r_{\mathrm{e}} / \delta x_{\mathrm{i}}\right)}{1+(\gamma / 2) \xi(d F / d \xi)\left(R_{B, \mathrm{~d}} / R_{B}\right)}\right\} \delta x_{\mathrm{i}} .
$$

Denoting $(\xi / F)(d F / d \xi)$ by $\eta$, Eq. (C11) is rewritten as

$$
\delta R_{B} \sim \frac{1+3\left(\delta r_{\mathrm{e}} / \delta x_{\mathrm{i}}\right) \eta\left(r_{\mathrm{e}} d \ln R_{B, \mathrm{~d}} / d x\right)^{-1}}{1+(\gamma / 2) \eta} F \frac{d R_{B, \mathrm{~d}}}{d x} \delta x_{\mathrm{i}} .
$$

Since $\delta r_{\mathrm{e}} / \delta x_{\mathrm{i}}$ is approximately equal to the mapping (between ionosphere and equator) factor in the latitudinal/radial dimension, $\left(\delta r_{\mathrm{e}} / \delta x_{\mathrm{i}}\right)\left(d \ln R_{B, \mathrm{~d}} / d x\right)^{-1}$ represents the characteristic scale length, on the equatorial plane, for the variation of $R_{B, \mathrm{~d}}$, which is denoted by $r_{R}$. Equation (C12) is finally reduced to

$$
\delta R_{B} \sim\left\{\frac{1+3 \eta\left(r_{R} / r_{\mathrm{e}}\right)}{1+(\gamma / 2) \eta} F \frac{d R_{B, \mathrm{~d}}}{d x}\right\} \delta x_{\mathrm{i}} .
$$

Using the magnetic field model by Tsyganenko (1987), for the region 1 zone we find that $\eta \sim 1$ although it weakly depends on latitude, and that $F$ and $r_{R} / r_{\mathrm{e}}$ increase with latitude, from about 2 to 30 and about 0.5 to 2.0 , respectively. Thus the value of the curly-bracketed factor in Eq. (C13) is greater, to more degree at higher latitude, compared with that of $d R_{B, \mathrm{~d}} / d x$, which is just the ratio of $\delta R_{B} / \delta x_{\mathrm{i}}$ for the dipole field under the electrostatic approximation. (The factor of $(\gamma / 2) \eta$, which represents the effect of the tail current intensification accompanying the inward plasma convection, tends to suppress the change $\left(\left|\delta R_{B}\right|\right)$ of the flux tube volume, although this effect is minor.)

Since $d R_{B, \mathrm{~d}} / d x$ is positive, we find that $R_{B}$ decreases with decreasing $x_{\mathrm{i}}$ (i.e., the inward convection), and that $\left|\delta R_{B}\right|$ increases with $\left|\delta x_{\mathrm{i}}\right|$. Since on the nightside, the curly-bracketed factor in Eq. (C13) will not greatly change with the local time, on the deformed $N$-contour as shown in Fig. 17 the flux tube volume $R_{B}$ nearer to midnight has a smaller value because of greater inward displacement $\left(-\delta x_{\mathrm{i}}\right)$, namely $R_{B}$ on the $N$-contour has azimuthal (away from midnight) gradients. From Eqs. (C1) and (7) (in text), we find that in the situation of Fig. 17 the region 1 FAC can be generated on the poleward side of the HPT, where the gradient of $\partial N / \partial x$ is positive. This fact means that the tail current disruption proposed in our paper can take place even when the intensification of the tail current by the adiabatic plasma compression as well as the associated change of the magnetic field are allowed to occur in the course of inward convection.

In the above discussion we do not consider the (feedback) effect of the tail current disruption on the variation of the magnetic field configuration. To represent this effect on the relation (C10) between $\delta B_{0}$ and $\delta R_{B}$, we can take the parameter $\gamma$ to be smaller in the region where the current disruption occurs (although $\gamma$ originally denotes the ratio of specific heats). Then the decrement $\left|\delta R_{B}\right|$ in Eq. (C11) is found to be greater in the disruption region, compared with that in case that no current disruption occurs, simply indicating a result of dipolarization of a magnetic flux tube. When on the nightside the current disruption proceeds further in an MLT sector nearer to midnight, as shown in our numerical simulations (see Fig. 7), the azimuthal (away from midnight) gradient of $R_{B}$ on the deformed $N$-contour (see Fig. 17) increases in magnitude due to the dipolarization caused by the current disruption, which results in intensification of the region 1 FACs, or equivalently further progression of the disruption. In summary it may be concluded that the tail current disruption as proposed in our paper can well proceed, under the influence of a two-cell convection, in the situation that the magnetic field configuration is allowed to change responding to variations of the tail current intensity. 


\section{Appendix D}

In this appendix we examine how deflection of the field lines caused by the field-aligned currents can affect the generation of FACs proposed in our paper. First note that a perturbation $(\delta \boldsymbol{B})$ of the magnetic field caused by the FACs is perpendicular to the current direction (i.e., field line direction), and that consequently the field line deflection occurs in a plane parallel to the current sheet (if the approximation of a "sheet current" is allowed) (e.g., Nishitani, 1992). It is assumed that on the duskside the FACs are distributed, on the ionosphere, as schematically shown in Fig. 18(a), where the direction of the $y$ axis is taken to be the same as that of the average magnetic drift velocity $\overline{\boldsymbol{V}}_{\mathrm{m}, \mathrm{i}}$ (defined in Section 2), and the $x$ axis is directed poleward; the velocity $\overline{\boldsymbol{V}}_{\mathrm{m}, \mathrm{i}}$ as well as the $y$ axis are defined on the basis of the unperturbed field lines, i.e., the magnetic field distribution without $\delta \boldsymbol{B}$. The boundaries between the different current regions make an angle $\theta$ (i.e., distortion angle defined in Section 3) with the $y$ axis. (In Fig. 18, for simplicity the latitudinal dependence of the distortion angle $\theta$ is neglected (i.e., $\theta$ is constant).) Hereafter, our discussion is focused on the FAC effect on the duskside in the northern hemisphere because extension to the dawnside or the southern hemisphere is straightforward. The magnetic field perturbation (or its projection to the ionosphere), $\delta \boldsymbol{B}$, which is caused by the FACs, is schematically illustrated in Fig. 18(a). (Needless to say, on a fixed field line $\delta B$ changes with the altitude. The plot of $\delta \boldsymbol{B}$ in this figure shows, only conceptually, the latitudinal dependence of $\delta \boldsymbol{B}$ at one representative (but arbitrary) altitude.) Since the intensity of the region 1 FAC is assumed to be greater than that of the region $2, \delta \boldsymbol{B}$ does not vanish at the poleward boundary (at a point " $\mathrm{P}_{1}$ " in Fig. 18(a)) of the region 1 zone. Instead, at a point " $\mathrm{P}_{0}$ " inside the region 1 zone, $\delta \boldsymbol{B}$ vanishes. The perturbation $\delta \boldsymbol{B}$ is peaked in magnitude at the interface $\left(\mathrm{P}_{2}\right)$ between the region 1 and region $2 \mathrm{FAC}$ zones. The equatorial end, $\mathrm{E}_{1}$, (i.e., intersection with the equatorial plane) of a perturbed field line is displaced from its original position, $\mathrm{E}_{0}$, namely the equatorial end of the

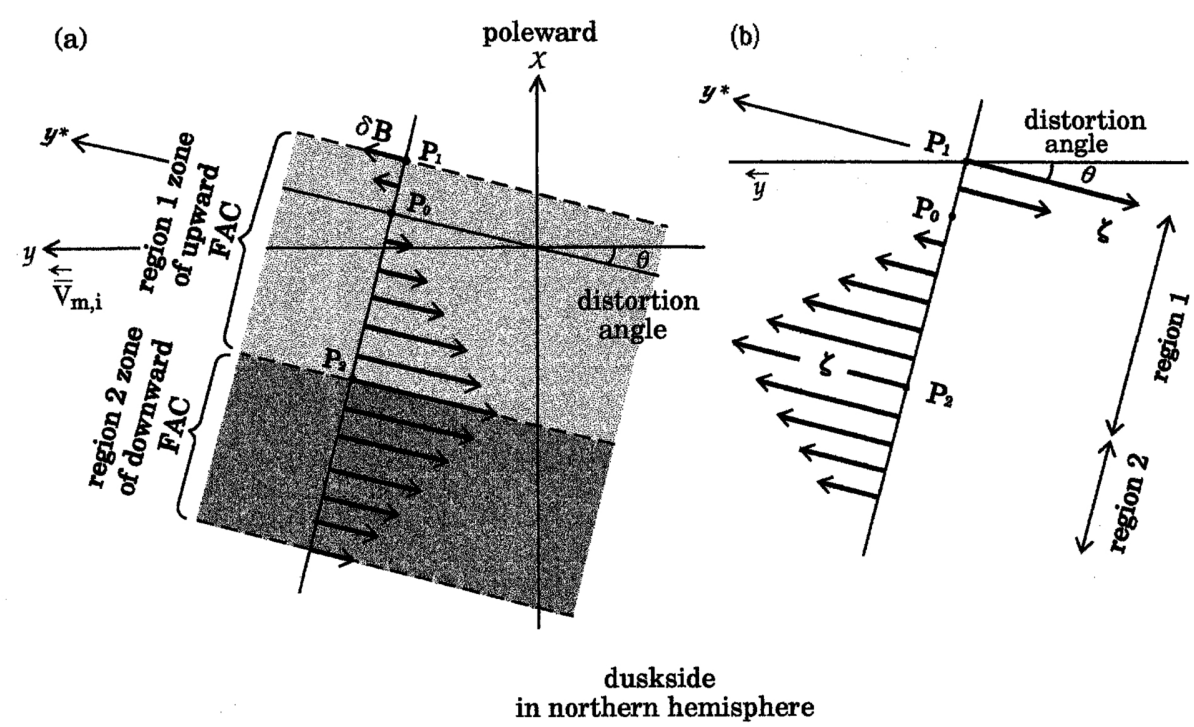

Fig. 18. Schematics showing (a) the magnetic field perturbation $\delta B$ at a certain altitude and (b) the corresponding displacement $\zeta$ of the field lines (defined in text), on the duskside in the northern hemisphere, which are caused by the region 1 and region 2 FACs. The direction of the $y^{*}$ axis at a point is defined as being parallel to the equicontour of the HPT flux tube content $N$ there. 
unperturbed field line with the same foot point, $\mathrm{G}_{0}$, on the ionosphere. Suppose that the point $E_{1}$ is mapped along the unperturbed field line to the point $G_{1}$ on the ionosphere. Then the vector $\overrightarrow{\mathrm{G}_{0} \mathrm{G}_{1}}$, which is denoted by $\zeta$, can represent the displacement (deflection) of the field line (with foot point at $\mathrm{G}_{0}$ ). (Note that $\overrightarrow{\mathrm{G}_{0} \mathrm{G}_{1}}$ is the ionospheric projection of the equatorial displacement $\overrightarrow{\mathrm{E}_{0} \mathrm{E}_{1}}$ of that field line.) The displacement $\zeta$ may be assumed to be parallel to the boundaries of the FAC zones, i.e., the $y^{*}$ axis indicated in Fig. 18. (The $y^{*}$ axis is locally defined, namely its direction at a point is defined as being parallel to the equicontour of the HPT flux tube content $N$ there.) Figure 18(b) schematically shows the latitudinal profile of the displacement $\zeta$ which corresponds to the $\delta \boldsymbol{B}$ distribution in Fig. 18(a).

According to the theoretical analysis by Nishitani (1992) and Donovan (1993), the displacement $|\zeta|$ of the field line foot point on the ionosphere can reach about $10^{\circ}$ in longitude (i.e., $\sim 350$ $\mathrm{km}$ in distance) at the interface between the region 1 and region 2 FAC zones where the perturbation $|\delta \boldsymbol{B}|$ is peaked. In the present calculation we assume that this large displacement occurs both at points $\mathrm{P}_{1}$ and $\mathrm{P}_{2}$ (see Fig. 18(b)). (Despite of the fact that $|\delta \boldsymbol{B}|$ at $\mathrm{P}_{2}$ is even smaller than that at $P_{1}$, such large displacement at $P_{1}$ may be possible for the following reason: the field lines with foot points at higher latitudes will be more easily deflected because the ambient (unperturbed) field strength at high altitudes can be smaller on the higher-latitude field lines.)

To begin with, we estimate the flux tube volume $R_{B}$ (defined in Section 2 ) in the situation that the field line deflection occurs due to the FACs. For the unperturbed field lines, the flux tube volume at a point $\mathrm{P}$ is denoted by $R_{B, 0}(\overrightarrow{\mathrm{OP}})$ where "O" is the origin of the ionospheric coordinates $(x, y)$. Within the order of magnitude, $R_{B}$ at $\mathrm{P}$ may be approximated by

$$
R_{B}(\overrightarrow{\mathrm{OP}}) \sim\left(R_{B, 0}(\overrightarrow{\mathrm{OP}}+\zeta)+R_{B, 0}(\overrightarrow{\mathrm{OP}})\right) / 2
$$

where $\boldsymbol{\zeta}$ is the above-defined displacement of the field line with foot point at $\mathrm{P}$. For the unperturbed magnetic field configuration, we define the characteristic scale length $\ell_{R}$ as $\ell_{R}=$ $\left(\partial \ln R_{B, 0} / \partial x\right)^{-1}$. Using the magnetic field model by Tsyganenko (1987), we find that $\ell_{R}$ is on the order of $100 \mathrm{~km}$ in the high-latitude part of the region 1 zone and greater than $100 \mathrm{~km}$ at lower latitudes. Noting that the $x$ component of $|\zeta|, \zeta_{x}$, is expressed as $\zeta_{x}= \pm|\zeta| \sin \theta$ and that $|\zeta| \lesssim 350 \mathrm{~km}$ and $\ell_{R} \gtrsim 100 \mathrm{~km}$, we find the ratio of $\zeta_{x} / \ell_{R}$ to be considerably smaller than unity for a small distortion angle satisfying $\sin \theta \lesssim 0.1$, which is the case with our numerical simulations. Because of $\partial R_{B, 0} / \partial y=0$ (see Eq. (C1)), expanding the rightside of Eq. (D1) with respect to the small parameter $\zeta_{x} / 2 \ell_{R}$, we obtain

$$
R_{B}(\overrightarrow{\mathrm{OP}}) \sim R_{B, 0}(\overrightarrow{\mathrm{OP}})+\frac{\zeta_{x}}{2} \frac{\partial R_{B, 0}(\overrightarrow{\mathrm{OP}})}{\partial x}=R_{B, 0}(\overrightarrow{\mathrm{OP}})\left(1+\frac{\zeta_{x}}{2 \ell_{R}}\right) .
$$

Now we evaluate the change in the field-aligned current density, which is caused by the effect of the field line deflection. From Eqs. (7) and (C1) the ratio between the current densities, $J_{\|, \mathrm{i}}$ and $J_{\|, \mathrm{i}, 0}$ for two cases of perturbed and unperturbed field lines is expressed as

$$
\frac{J_{\|, \mathrm{i}}}{J_{\|, \mathrm{i}, 0}}=\frac{\left(\ln R_{B} \times \nabla N\right) \cdot \boldsymbol{B}_{\mathrm{i}}}{\left(\ln R_{B, 0} \times \nabla N\right) \cdot \boldsymbol{B}_{\mathrm{i}}}=\frac{\partial \ln R_{B} / \partial y^{*}}{\partial \ln R_{B, 0} / \partial y^{*}}
$$

where, as defined above, $y^{*}$ is the distance along the equicontour of the HPT flux tube content $N$. The displacement $\zeta_{x}$ can be assumed independent of $y^{*}$, and $R_{B, 0}$ and $\ell_{R}$ are functions of only $x$ (being independent of $y$ ). Then, substituting Eq. (D2) into Eq. (D3) and noting $\partial / \partial y^{*}=\sin \theta \partial / \partial x$ for $R_{B, 0}(x)$ and $\ell_{R}(x)$, we obtain

$$
\frac{J_{\|, \mathrm{i}}}{J_{\|, \mathrm{i}, 0}} \sim 1-\frac{\zeta_{x}}{2} \frac{\partial \ln \ell_{R} / \partial x}{1+\zeta_{x} / 2 \ell_{R}} \sim 1-\frac{\zeta_{x}}{2} \frac{\partial}{\partial x} \ln \ell_{R}
$$


On the basis of the Tsyganenko's $(1987,1989)$ model, it is assumed that $\partial \ln \ell_{R} / \partial x$ is negative in the region 1 and region 2 FAC zones, and that the scale length of $\left|\partial \ln \ell_{R} / \partial x\right|^{-1}$ is the same order of magnitude as $\ell_{R}(\sim 100 \mathrm{~km})$ in the high-latitude part of the region 1 zone, but it is even greater at lower latitudes. Therefore, from Eq. (D4) we find the followings: 1 . In the high-latitude part of the region 1 zone where $\zeta_{x}$ is negative (say, in the interval between $\mathrm{P}_{0}$ and $\mathrm{P}_{1}$ in Fig. 18), $\left|J_{\|, \mathrm{i}}\right|$ is decreased by the effect of the field line deflection; the decrement may reach about $20 \%$ of $\left|J_{\|, \mathrm{i}, 0}\right|$, for $\sin \theta \lesssim 0.1$ and $|\zeta| \lesssim 350 \mathrm{~km}$, at the poleward edge of the region 1 zone. 2 . In the other part $\left(\overline{\mathrm{P}_{0} \mathrm{P}_{2}}\right.$ in Fig. 18) of the region 1 zone as well as the region 2 zone where $\zeta_{x}$ is positive, $J_{\|, \mathrm{i}}$ is increased, but the increment is less than $5 \%$ if the scale length of $\left|\partial \ln \ell_{R} / \partial x\right|^{-1}>400 \mathrm{~km}$ near the region 1 /region 2 interface is assumed. 3 . The above-described changes of $J_{\|, \mathrm{i}}$ is independent of whether the location is on the duskside or dawnside and in the northern or southern hemisphere. (Note that $\boldsymbol{\zeta}$ is parallel to $\delta \boldsymbol{B}$ in the southern hemisphere while $\boldsymbol{\zeta}$ is antiparallel to $\delta \boldsymbol{B}$ in the northern hemisphere.)

\section{Appendix E}

In this appendix we briefly describe the theoretical model proposed by Yamamoto and Ozaki (1994), which attempts to explain the persistent existence of the region 1 and region 2 field-aligned currents as well as the potential drop across the polar cap after the interplanetary magnetic field turns northward. Important concepts in the model are 1. the field-aligned current (FAC) generation due to the HPT distortion by the two-cell convection (see text), and 2 . the neutral wind "flywheel" as a result of the thermosphere-magnetosphere coupling (Lyons et al., 1985).

During the periods of southward IMF, the two-cell convection maintained by the combined effects of the solar wind voltage and the HPT polarization can affect the high-latitude, neutral particle circulation in the thermosphere through the ion drag mechanism: at $\mathrm{F}$ region altitudes the convecting ions impart momentum to the neutral particles through ion drag forcing, which causes the neutral winds to adopt a circulation pattern similar to the ion convection pattern. Development of the neutral gas vorticity, $\Omega$, is described, as a first approximation (Thayer and Killeen, 1993), by the equation of $\partial \Omega / \partial t \sim \alpha\left(\Omega_{\mathrm{I}}-\Omega\right)$, where $\Omega_{\mathrm{I}}$ is the vorticity of the ion convection, and $\alpha$ is the ion-drag parameter given as $\alpha=\sigma_{\mathrm{P}} B^{2} / \rho_{\mathrm{m}} ; \sigma_{\mathrm{P}}$ is the Pedersen conductivity, $B$ is the magnetic field intensity, and $\rho_{\mathrm{m}}$ is the neutral gas (mass) density. The required time for the neutral vorticity evolution to $\Omega \sim \Omega_{\mathrm{I}}$ is given by $\alpha^{-1}$, which depends strongly on the altitude: for example, $\alpha^{-1} \sim 30 \mathrm{~min}$ at $300 \mathrm{~km}$ altitude and $\alpha^{-1} \sim 6$ hours at $200 \mathrm{~km}$. Consequently, the longer time period of a magnetic storm with IMF $B_{Z}<0$ leads to creation of the two-cell circulation of the neutral winds at lower altitudes. The polarization electric field $\boldsymbol{E}_{\mathrm{N}}$ is expected to arise from the neutral wind dynamo; $\boldsymbol{E}_{\mathrm{N}}=-\Sigma_{\mathrm{P}}^{-1} \int d h \sigma_{\mathrm{P}}\left(\boldsymbol{U}_{\mathrm{N}} \times \boldsymbol{B}\right)$, where $\boldsymbol{U}_{\mathrm{N}}$ is the neutral wind velocity, $\Sigma_{\mathbf{P}}$ is the height-integrated Pedersen conductivity, and $h$ is the altitude. As the storm is prolonged in time, the electric potential from the neutral wind dynamo comes to significantly contribute to the polar cap potential drop.

After the IMF $B_{Z}$ turns northward, the solar wind convection does not work because the field lines emanating from the polar cap are probably closed, losing the direct connection with the solar wind. Instead, the two-cell convection caused by the neutral wind dynamo could act to deform the HPT in such a manner as generating the paired region $1 /$ region 2 FACs. Since the polar cap potential drop, $\Phi_{\mathrm{N}}$, of neutral wind origin is assumed to decrease with a time constant of $\alpha^{-1}$ (estimated at the lowest altitude where the neutral circulation is well developed), the potential drop, $\Phi_{R 1,2}$, associated with the region 1 /region 2 FACs and consequently the total potential drop, $\Phi_{R 1,2}+\Phi_{\mathrm{N}}$, will also decay with the same characteristic time. This prediction agrees with the observations of exponential decay of the polar cap potential after the northward turning of the IMF, which was obtained by Wygant et al. (1983) (see Fig. 11 in our text). Thus 
we can understand that the paired region 1/region 2 FACs can endure for many hours (while decaying) after a magnetic storm, as a result of the HPT polarization under the influence of the long-lived (although decaying) two-cell convection of neutral wind origin.

\section{REFERENCES}

Axford, W. I. and C. O. Hines, A unifying theory of high-latitude geophysical phenomena and geomagnetic storms, Can. J. Phys., 39, 1433-1464, 1961.

Block, L. P. and C.-G. Fälthammar, The role of magnetic-field-aligned electric fields in auroral acceleration, $J$. Geophys. Res., 95, 5877-5888, 1990.

Cattell, C. A. and F. S. Mozer, Substorm electric fields in the earth's magnetotail, in Magnetic Reconnection in Space and Laboratory Plasmas, Geophys. Monogr. Ser., vol. 30, edited by E. Hones, Jr., pp. 208-215, AGU, Washington, D.C., 1984.

Chmyrev, V. M., V. N. Oraevsky, S. V. Bilichenko, N. V. Isaev, G. A. Stanev, D. K. Teodosiev, and S. I. Shkolnikova, The fine structure of intense small-scale electric and magnetic fields in the high-latitude ionosphere as observed by Intercosmos Bulgaria 1300 satellite, Planet. Space Sci., 33, 1383-1388, 1985.

Donovan, E. F., Modeling the magnetic effects of field-aligned currents, J. Geophys. Res., 98, 13529-13543, 1993.

Dubinin, E. M., P. L. Israelevich, N. S. Nikolaeva, I. Kutiev, and I. M. Podgorny, Localized auroral disturbances in the morning sector of topside ionosphere as a standing electromagnetic wave, Planet. Space Sci., 33, 597-606, 1985.

Dubinin, E. M., A. S. Volokitin, P. L. Israelevich, and N. S. Nokolaeva, Auroral electromagnetic disturbances at altitudes of $900 \mathrm{~km}$ : Alfvén wave turbulence, Planet. Space Sci., 36, 949-962, 1988.

Dungey, J. W., Interplanetary field and the auroral zones, Phys. Rev. Lett., 6, 47-48, 1961.

Eastman, T. E. and E. W. Hones, Jr., Characteristics of the magnetospheric boundary layer and magnetopause layer as observed by Imp 6, J. Geophys. Res., 84, 2019-2028, 1979.

Friis-Christensen, E. and K. Lassen, Large-scale distribution of discrete auroras and field-aligned currents, in Auroral Physics, edited by C.-I. Meng, M. J. Rycroft, and L. A. Frank, pp. 369-381, Cambridge UP, 1991.

Fujii, R. and T. Iijima, Control of the ionospheric conductivities on large-scale Birkeland current intensities under geomagnetic quiet conditions, J. Geophys. Res., 92, 4505-4513, 1987.

Gurnett, D. A. and L. A. Frank, A region of intense plasma wave turbulence on auroral field lines, J. Geophys. Res., 82, 1031-1050, 1977.

Gurnett, D. A., L. A. Frank, and R. P. Lepping, Plasma waves in the distant magnetotail, J. Geophys. Res., 81, 6059-6071, 1976.

Gurnett, D. A., R. L. Huff, J. D. Menietti, J. L. Burch, J. D. Winningham, and S. D. Shawhan, Correlated low-frequency electric and magnetic noise along the auroral field lines, J. Geophys. Res., 89, 8971-8985, 1984.

Hardy, D. A., M. S. Gussenhoven, F. J. Rich, and D. H. Brautigam, The average pattern of auroral particle precipitation, its associated conductivity and field aligned currents, J. Geomag. Geoelectr., 43, Suppl., 337351, 1991.

Harel, M., R. A. Wolf, P. H. Reiff, R. W. Spiro, W. J. Burke, F. J. Rich, and M. Smiddy, Quantitative simulation of a magnetospheric substorm, 1. Model logic and overview, J. Geophys. Res., 86, 2217-2241, 1981a.

Harel, M., R. A. Wolf, R. W. Spiro, P. H. Reiff, C.-K. Chen, W. J. Burke, F. J. Rich, and M. Smiddy, Quantitative simulation of a magnetospheric substorm, 2. Comparison with observations, J. Geophys. Res., 86, 2242-2260, 1981b.

Iijima, T. and T. A. Potemra, The amplitude distribution of field-aligned currents at northern high latitudes observed by Triad, J. Geophys. Res., 81, 2165-2174, 1976.

Iijima, T. and T. A. Potemra, Large-scale characteristics of field-aligned currents associated with substorms, J. Geophys. Res., 83, 599-615, 1978.

Iijima, T., M. Watanabe, T. A. Potemra, L. J. Zanetti, and F. J. Rich, The relationship between ion and electron precipitation patterns and field-aligned current systems during a substorm, in Magnetospheric Substorms, Geophys. Monogr. Ser., vol. 64, edited by J. R. Kan et al., pp. 97-110, AGU, Washington, D.C., 1991.

Iijima, T., T. A. Potemra, L. J. Zanetti, and S. Ohtani, Distribution of particle and Birkeland currents: IMF dependent north-south conjugacy (abstracts), paper presented at Society of Geomagnetism and Earth, Planetary and Space Sci., Tokyo, October, 1992.

Kamide, Y. and W. Baumjohann, Estimation of electric fields and currents from International Magnetospheric Study magnetometer data for the CDAW 6 intervals: implications for substorm dynamics, J. Geophys. Res., 90, 1305-1317, 1985.

Kamide, Y., Y. Ishihara, T. L. Killeen, J. D. Craven, L. A. Frank, and R. A. Heelis, Combining electric field and aurora observations from DE 1 and 2 with ground magnetometer records to estimate ionospheric electromagnetic quantities, J. Geophys. Res., 94, 6723-6738, 1989.

Karty, J. L., R. A. Wolf, and R. W. Spiro, Region one currents connecting to sunward convecting flux tubes, in Magnetospheric Currents, Geophys. Monogr. Ser., vol. 28, edited by T. A. Potemra, pp. 269-275, AGU, Washington, D.C., 1984. 
Lee, W. W. and H. Okuda, A simulation model for studying low frequency microinstabilities, J. Comput. Phys., 26, 139-152, 1978.

Lundin, R., G. Gustafsson, A. I. Eriksson, and G. Marklund, On the importance of high-altitude low-frequency electric fluctuations for the escape of ionospheric ions, J. Geophys. Res., 95, 5905-5919, 1990.

Lyons, L. R., A simple model for polar cap convection patterns and generation of $\theta$ auroras, J. Geophys. Res., 90, 1561-1567, 1985.

Lyons, L. R. and T. W. Speiser, Evidence for current sheet acceleration in the geomagnetic tail, J. Geophys. Res., 87, 2276-2286, 1982.

Lyons, L. R., T. L. Killeen, and R. L. Walterscheid, The neutral wind "flywheel" as a source of quiet-time, polar-cap currents, Geophys. Res. Lett., 12, 101-104, 1985.

McDiarmid, I. B., J. R. Burrows, and E. E. Budzinski, Average characteristics of magnetospheric electrons (150 $\mathrm{eV}$ to $200 \mathrm{keV}$ ) at $1400 \mathrm{~km}, J$. Geophys. Res., 80, 73-79, 1975.

Nagai, T., Observed magnetic substorm signatures at synchronous altitude, J. Geophys. Res., 87, 4405-4417, 1982.

Nishitani, N., Magnetic field line connection between the ionosphere and the magnetosphere-Auroral activity and relevant magnetic field variations at geosynchronous orbit, Ph.D. Thesis for Nagoya Univ., 1992.

Ohtani, S., S. Kokubun, R. Nakamura, R. C. Elphic, C. T. Russell, and D. N. Baker, Field-aligned current signatures in the near-tail region 2. Coupling between the region 1 and the region 2 systems, J. Geophys. Res., 95, 18913-18927, 1990.

Okuda, H., Introduction to particle simulation models and other application to electrostatic plasma waves in space, in Computer Simulation of Space Plasmas, edited by H. Matsumoto and T. Sato, pp. 3-41, Terra Scientific, Tokyo and Reidel, Dordrecht, 1985.

Piddington, J. H., The magnetospheric radiation belt and tail plasma sheet, Planet. Space Sci., 16, 703-716, 1968.

Richmond, A. D., Y. Kamide, B.-H. Ahn, S.-I. Akasofu, D. Alcaydé, M. Blanc, O. de la Beaujardiére, D. S. Evans, J. C. Foster, E. Friis-Christensen, T. J. Fuller-Rowell, J. M. Holt, D. Knipp, H. W. Kroehl, R. P. Lepping, R. J. Pellinen, C. Senior, and A. N. Zaitev, Mapping electrodynamics features of the high-latitude ionosphere from localized observations: combined incoherent-scatter radar and magnetometer measurements for January 18-19, 1984, J. Geophys. Res., 93, 5760-5776, 1988.

Rostoker, G. and R. Boström, A mechanism for driving the gross Birkeland current configuration in the auroral oval, J. Geophys. Res., 81, 235-244, 1976.

Saito, Y., T. Mukai, M. Hirahara, S. Machida, and N. Kaya, Distribution function of precipitating ion beams with velocity dispersion observed near the poleward edge of the nightside auroral oval, Geophys. Res. Lett., 19, 2155-2158, 1992.

Sato, T., Strong plasma acceleration by slow shocks resulting from magnetic reconnection, J. Geophys. Res., 84, 7177-7190, 1979.

Sato, T., Auroral physics, in Magnetospheric Plasma Physics, edited by A. Nishida, pp. 197-243, D. Reidel, Norwell, Mass., 1982.

Sato, T., H. Matsumoto, and K. Nagai, Particle acceleration in time-developing magnetic reconnection process, $J$. Geophys. Res., 87, 6089-6097, 1982.

Scarf, F., L. Frank, K. Ackerson, and R. Lepping, Plasma wave turbulence at distant crossings of the plasma sheet boundaries and neutral sheet, Geophys. Res. Lett., 1, 189-192, 1974.

Sonnerup, B. U. Ö., Theory of the low-latitude boundary layer, J. Geophys. Res., 85, 2017-2026, 1980.

Stern, D. P., Magnetospheric dynamo processes, in Magnetospheric Currents, Geophys. Monogr. Ser., vol. 29, edited by T. A. Potemra, pp. 200-207, AGU, Washington, D.C., 1983.

Thayer, J. P. and T. L. Killeen, A kinematic analysis of the high-latitude thermospheric neutral circulation pattern, J. Geophys. Res., 98, 11549-11565, 1993.

Tsyganenko, N. A., Global quantitative models of the geomagnetic field in the cislunar magnetosphere for different disturbance levels, Planet. Space Sci., 35, 1347-1358, 1987.

Tsyganenko, N. A., A magnetospheric magnetic field model with a warped tail current sheet, Planet. Space Sci., 37, 5-20, 1989.

Watanabe, M. and T. Iijima, Substorm growth phase on the magnetotail, J. Geophys. Res., 98, 17299-17316, 1993.

Woch, J. and R. Lundin, The low-latitude boundary layer at mid-altitudes: identification based on Viking hot plasma data, Geophys. Res. Lett., 20, 979-982, 1993.

Woch, J., M. Yamauchi, and R. Lundin, The low-latitude boundary layer at mid-altitudes: relation to large-scale Birkeland currents, Geophys. Res. Lett., 20, 2251-2254, 1993.

Wolf, R. A., The quasi-static (slow-flow) region of the magnetosphere, in Solar-Terrestrial Physics, edited by R. L. Carovillano and J. M. Forbes, pp. 303-368, D. Reidel, Hingham, Mass., 1983.

Wygant, J. R., R. B. Torbert, and F. S. Mozer, Comparison of S3-3 polar cap potential drops with the interplanetary magnetic field and models of magnetopause reconnection, J. Geophys. Res., 88, 5727-5735, 1983.

Yamamoto, T. and M. Ozaki, A new model of the field-aligned current generation after a geomagnetic storm (abstracts), paper presented at Society of Geomagnetism and Earth, Planetary and Space Sci., Sendai, March, 
1994.

Yamamoto, T., K. Makita, M. Ozaki, and C.-I. Meng, A particle simulation of auroral omega bands and torch-like structures, J. Geomag. Geoelectr., 45, 619-648, 1993.

Yamamoto, T., S. Inoue, N. Nishitani, M. Ozaki, and C.-I. Meng, A theory for generation of the paired region 1 and region 2 field-aligned currents, J. Geophys. Res., 101, 27199-27222, 1996.

Yamamoto, T., S. Inoue, and C.-I. Meng, Formation of auroral omega bands in the paired region 1 and region 2 field-aligned current system, J. Geophys. Res., 102, 2531-2544, 1997a.

Yamamoto, T., S. Inoue, and C.-I. Meng, Effect of anomalous cross-field diffusion on the field-aligned current generation, J. Geomag. Geoelectr., 49, this issue, 923-945, $1997 \mathrm{~b}$.

Yang, Y. S., R. W. Spiro, and R. A. Wolf, Generation of region 1 current by magnetospheric pressure gradients, J. Geophys. Res., 99, 223-234, 1994. 